\title{
Analytical Profile and Antioxidant and Anti-Inflammatory Activities of the Enriched Polyphenol Fractions Isolated from Bergamot Fruit and Leave
}

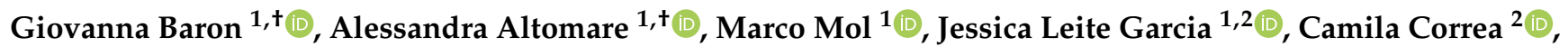 \\ Angela Raucci ${ }^{3}$, Luigi Mancinelli $^{3}$, Sarah Mazzotta ${ }^{1}{ }^{1}$, Laura Fumagalli ${ }^{1}{ }^{(0}$, Giuseppe Trunfio ${ }^{4}$, Luigi Tucci ${ }^{4}$, \\ Elena Lombardo ${ }^{4}$, Domenico Malara ${ }^{4}$, Elzbieta Janda ${ }^{5}{ }^{\mathbb{D}}$, Vincenzo Mollace ${ }^{5}$, Marina Carini ${ }^{1}$, Ezio Bombardelli ${ }^{6}$ \\ and Giancarlo Aldini ${ }^{1, *(1)}$
}

check for updates

Citation: Baron, G.; Altomare, A.; Mol, M.; Garcia, J.L.; Correa, C.; Raucci, A.; Mancinelli, L.; Mazzotta, S.; Fumagalli, L.; Trunfio, G.; et al. Analytical Profile and Antioxidant and Anti-Inflammatory Activities of the Enriched Polyphenol Fractions Isolated from Bergamot Fruit and Leave. Antioxidants 2021, 10, 141. https://doi.org/10.3390/ antiox10020141

Received: 11 December 2020

Accepted: 15 January 2021

Published: 20 January 2021

Publisher's Note: MDPI stays neutral with regard to jurisdictional claims in published maps and institutional affiliations.

Copyright: (c) 2021 by the authors. Licensee MDPI, Basel, Switzerland. This article is an open access article distributed under the terms and conditions of the Creative Commons Attribution (CC BY) license (https:// creativecommons.org/licenses/by/ $4.0 /)$.
1 Department of Pharmaceutical Sciences, University of Milan, 20133 Milan, Italy; giovanna.baron@unimi.it (G.B.); alessandra.altomare@unimi.it (A.A.); marcomol89@gmail.com (M.M.); jessleitegarcia@gmail.com (J.L.G.); sarah.mazzotta@unimi.it (S.M.); laura.fumagalli@unimi.it (L.F.); marina.carini@unimi.it (M.C.)

2 Medical School, Sao Paulo State University (Unesp), Botucatu 18618-687, Brazil; correa.camila9@gmail.com

3 Experimental Cardio-oncology and Cardiovascular Aging Unit, Centro Cardiologico Monzino-IRCCS, Via Carlo Parea, 4, 20138 Milan, Italy; angela.raucci@cardiologicomonzino.it (A.R.); jkducati695@gmail.com (L.M.)

4 H\&AD Srl, 89032 Bianco, Italy; g.trunfio@head-sa.com (G.T.); 1.tucci@head-sa.com (L.T.); e.lombardo@head-sa.com (E.L.); d.malara@head-sa.com (D.M.)

5 Department of Health Sciences, University "Magna Graecia” of Catanzaro, 88100 Catanzaro, Italy; janda@unicz.it (E.J.); mollacev@gmail.com (V.M.)

6 Plantexresearch Srl, 20122 Milan, Italy; ezio.bombardelli@plantexresearch.it

* Correspondence: giancarlo.aldini@unimi.it; Tel.: +39-02-503-19296

† Giovanna Baron and Alessandra Altomare contributed equally to this work.

Abstract: The aim of the study is to compare the qualitative and semi-quantitative profile of the polyphenol fraction purified from the leaf (BLPF) and fruit (BFPF) of bergamot (Citrus bergamia), and to evaluate their antioxidant and anti-inflammatory activity. The analytical qualitative profile was carried out by LC-ESI/MS using three different approaches: targeted (searching analytes already reported in bergamot extract), semi-targeted (a selective search of 3-hydroxy-3-methylglutarate [HMG] derivatives involved in the cholesterol reducing activity of BPF) and untargeted. A total number of 108 compounds were identified by using the three approaches, 100 of which are present in both the extracts thus demonstrating a good qualitative overlapping of polyphenols between the two extracts. The antioxidant activity was higher for BLPF in respect to BFPF but when normalized in respect to the polyphenol content they were almost overlapping. Both the extracts were found to dose dependently inhibit cell inflammation stimulated with IL-1 $\alpha$. In conclusion, the comparison of the qualitative and quantitative profile of polyphenols as well as of the antioxidant and anti-inflammatory activity of bergamot leaf and fruit well indicates that leaf is a valid source of bergamot polyphenol extraction and an even richer source of polyphenol in respect to the fruit.

Keywords: Citrus bergamia; polyphenols; high-resolution mass spectrometry; antioxidant; inflammation; metabolic syndrome; cholesterol; HMG

\section{Introduction}

The bergamot (Citrus bergamia), is an ancient fruit bearing tree used for the production of its essential oil. Although native to South-East Asia, the plant is also grown in Italy where due to the fact that it is very sensitive to the pedoclimatic conditions of the soil, bergamot cultivation is currently limited to the coastal area of Calabria (southern Italy), from Reggio Calabria to Locri, where the climate and environmental conditions are favorable to its 
cultivation. Italy accounts for over 95\% of world production of bergamot essential oil which is obtained from the peel and is widely used in the cosmetics industry [1-3].

Besides the commercial and scientific interest in the essential oil, in recent years the scientific community has focused growing attention on the juice of bergamot which has interesting nutraceutical potential as reviewed in some recent papers [4-6]. In particular, several pharmacological and intervention studies have reported that bergamot juice or rather its enriched polyphenolic fraction (BPF), obtained from the peeled fruit and mainly composed of flavanones (such as naringenin, hesperetin, eriodictyol glycosides), flavones, (apigenin, luteolin, chrysoeriol, diosmetin glycosides) and their 3-hydroxy-3-methyl-glutaryl (HMG) derivatives, has hypolipemic, hypoglycemic, and anti-inflammatory activities and, more generally, is effective in the treatment of metabolic syndrome symptoms [7-10]. Such in vivo effects have been linked to the antioxidant and anti-inflammatory activities of its constituents and to the ability of HMG derivatives, such as melitidin and brutieridin, to bind the catalytic site of HMG-CoA reductase and inhibit cholesterol synthesis by replacing its endogenous substrate HMG-CoA [11,12].

Based on the growing scientific evidence of the positive effects of BPF on human health, we believe that in the next few years there will be an increased demand for BPF as a supplementary ingredient. However, considering the limited availability of the fruit due to the restricted area of bergamot cultivation, the increased demand for the bergamot polyphenols should be addressed by considering an alternative source which must have a qualitative and quantitative composition and biological activity overlapping those of the fruit.

Analytical studies show that for some plants, such as some of those which produce berries, the phenolic composition of the leaf is similar to that of the precious fruit or even richer and higher, indicating that they may be utilized as an alternative source of bioactive natural products for the development of food supplements, nutraceuticals, or functional foods [13].

The aim of the paper is to fully analyze and compare the qualitative and semiquantitative profile of enriched polyphenol fraction from bergamot leaf (BLPF) and fruit (BFPF) and prepared by using the same process so that their qualitative and quantitative profile can be compared. The two extracts would then be evaluated in terms of antioxidant and anti-inflammatory activity. The results will permit us to understand whether bergamot leaves can be considered as an alternative plant source to the fruit for the extraction of polyphenols to be used as food supplements and nutraceuticals.

\section{Materials and Methods}

\subsection{Reagents}

6-hydroxy-2,5,7,8-tetramethyl-3,4-dihydrochromene-2-carboxylic acid (Trolox, cat. number 238813), DMSO (cat. number 472301), formic acid (cat. number 00940), ammonium acetate (cat. number 238074), Sephadex ${ }^{\mathrm{TM}}$ LH-20 (cat. number GE17-0090), bradykinin acetate (cat. number B3259), tannic acid (cat. number 403040), Folin-Ciocalteu reagent (cat. number 47641), sodium carbonate (cat. number 223530), gallic acid (cat. number G7384), 2',7'-dichlorofluorescein (cat. number 410217), sodium phosphate dibasic (cat. number S9763), sodium phosphate monobasic monohydrate (cat. number S9638), 2,2'-azobis(2-methylpropionamidine) dihydrochloride (ABAP, cat. number 440914), 2,2'azino-bis (3-ethylbenzothiazoline-6-sulfonic acid) diammonium salt (ABTS, cat. number A1888), potassium persulfate (cat. number 216224), sodium acetate (cat. number 241245), acetic acid (cat. number 695092), 2,2-diphenyl-1-picrylhydrazyl (DPPH, cat. number D9132), 3-(4,5-Dimethyl-2-thiazolyl)-2,5-diphenyl-2H-tetrazolium bromide (MTT, cat. number M2128), IL-1 $\alpha$ (cat. number SRP3310) and LC-MS grade solvents were purchased from Merck KGaA, Darmstadt, Germany. The peptide LVNEVTEF was custom synthesized by Sigma-Aldrich (Milan, Italy). LC-grade $\mathrm{H}_{2} \mathrm{O}\left(18 \mathrm{M} \Omega \mathrm{cm}\right.$ ) was prepared with a Milli-Q $\mathrm{H}_{2} \mathrm{O}$ purification system (Millipore, Bedford, MA, USA). 


\subsection{Plant Material}

\subsubsection{BFPF Preparation}

The albedo of bergamot fruit is minced with water in order to extract polyphenols, and to this the mixture is added a pectolytic enzyme to decrease the viscosity by degrading the pectin. The fluid is then clarified by means of an ultrafiltration process with semipermeable membranes having a selectivity equal to $12,000 \mathrm{Da}$. The solution is then passed through a polystirenic absorbing resin bed with pores of 100-150 Angstroms diameter. The entrapped polyphenols are then eluted by modifying their external conformation making the $\mathrm{pH}$ basic. Since the polyphenols in this form are unstable, they are passed through a cationic resin bed to re-establish the natural acid $\mathrm{pH}$ of 2.0-3.0. The obtained water solution is evaporated under vacuum at temperatures up to $60^{\circ} \mathrm{C}$ for less than $1 \mathrm{~min}$ until a concentrated water solution with a total polyphenol concentration of $40 \%$ is obtained. This is then dried in a spray dryer system thus obtaining a powder with less than $4.0 \%$ of humidity.

\subsubsection{BLPF Preparation}

The bergamot leaves are harvested and subsequently minced and a water/ethanol $(30 / 70, \% v / v)$ solution is added to the extract the polyphenols. The ethanol is then distilled, and the clarified water solution is passed through a polystirenic absorbing resin bed having with pores of 100-150 Angstroms diameter. The entrapped polyphenols are then eluted with pure ethanol. The obtained ethanolic solution is distilled at temperatures up to $40{ }^{\circ} \mathrm{C}$ obtaining a concentrated containing residual water with polyphenols, which is then dried in a spray dryer system obtaining a powder with less than $4.0 \%$ of humidity.

\subsection{LC-HR-MS Conditions}

The stock solutions $(2.5 \mathrm{mg} / \mathrm{mL})$ of the two bergamot extracts were prepared by dissolving the powder in methanol, then diluted 1:4 in $\mathrm{H}_{2} \mathrm{O} / \mathrm{HCOOH}, 100 / 0.1, \% v / v$ (mobile phase A) and spiked with the internal standard (6-hydroxy-2,5,7,8-tetramethyl3,4-dihydrochromene-2-carboxylic acid) at a final concentration of $50 \mu \mathrm{M}$. Each sample $(20 \mu \mathrm{L})$ was analyzed in triplicate by LC-HRMS as described by Baron et al. with few modifications [14]. Briefly, the chromatographic separation was performed using an RP Agilent Zorbax SB-C18 column $(150 \times 2.1 \mathrm{~mm}$, i.d. $3.5 \mu \mathrm{m}$, CPS analitica, Milan, Italy $)$ by an UltiMate 3000 system (Dionex) with a multistep program $(80 \mathrm{~min}$ ) of mobile phase A $\left(\mathrm{H}_{2} \mathrm{O} / \mathrm{HCOOH}, 100 / 0.1, \% v / v\right)$ and $\mathrm{B}\left(\mathrm{CH}_{3} \mathrm{CN} / \mathrm{HCOOH}, 100 / 0.1, \% v / v\right)$. An LTQ Orbitrap XL mass spectrometer equipped with an ESI source was used as analyzer, working in data dependent scan mode: three different collision energies (CID) 10, 20 and $40 \mathrm{eV}$ were used to fragment each ion selected in the full MS scan to obtain the best fragmentation pattern for each type of metabolite. The spectra were acquired in negative ion mode. Xcalibur 4.0 and Chromeleon Xpress 6.80 were used for instrument control and spectra analysis.

\subsection{Targeted, Semi-Targeted and Untargeted Analysis of Bergamot Extract Components}

A database (Table S1 of Supplementary Materials) was built searching in the literature for the known bergamot components [9,15-25]. The targeted analysis was performed by searching for all the components listed in the database on the basis of their exact mass $\left([\mathrm{M}-\mathrm{H}]^{-}\right)$, with a mass tolerance of $5 \mathrm{ppm}$. The fragmentation pattern was used to confirm the identity. A semi-targeted approach was designed to identify the most intense HMG derivatives according to the peculiar mass losses of 3-hydroxy-3-methylglutarate (HMG): three ion maps were generated on the Qual Browser of Xcalibur by selecting the three neutral losses: 62,102 and $144 \mathrm{Da}$, with a mass tolerance of $0.3 \mathrm{Da}$. Three lists of precursor ions were obtained and cross-checked to select the common ions. The fragmentation of the precursor ions was then manually verified to confirm the presence of the three losses. The hypotheses of identification were performed by using the Qual Browser Elemental Composition tool of Xcalibur with the following settings: charge -1 , mass tolerance 5 ppm, elements in use $\mathrm{C}<60, \mathrm{H}<100, \mathrm{O}<40, \mathrm{~N}<2$. The formulae thus obtained were compared to that of the structure hypothesized by the fragmentation. The 
untargeted analysis was performed by searching for the ions (intensity $>10^{4}$ counts) not identified using the previous methods. The top 5 molecular formulae calculated as for the semi-targeted approach were searched for in the available databases (HMDB, MoNa, PubChem, etc) to find candidates. The experimental MS/MS spectra were compared to those generated in silico by the Peak Assignment tool of CFM-ID online software to verify the identity (fragment tolerance $0.3 \mathrm{Da}$ ). The similarity search tool of MoNa was also used to annotate the unknown compounds as well as the Compound Identification tool of CFM-ID, using as mass tolerance $5 \mathrm{ppm}$ for the precursor ion and $0.3 \mathrm{Da}$ for the fragments. Finally, some losses were used to hypothesize structures not identified with the previous methods and were then confirmed by the Peak Assignment tool of CFM-ID: -162 for O-glucoside, -120 for C-glucoside, -146 for O-rhamnoside, -42 for acetyl moiety.

\subsection{Semi-Quantitative Data Analysis}

A semi-quantitative analysis of each identified metabolite was carried out by reconstituting the corresponding single ion chromatogram (SIC) by setting the molecular ion as filter ion and a tolerance of $5 \mathrm{ppm}$. The area under the curve of each metabolite was then automatically integrated as was that of the internal standard (Trolox). The ratio between the AUC of each metabolite $\left(A U C_{n}\right)$ and the AUC of the IS (AUC IS) was then calculated and divided by the sum of the ratios of all the compounds and expressed as $\%$ as reported by the equation (1)

$$
\left(\frac{\frac{\mathrm{AUC}_{\mathrm{n}}}{\mathrm{AUC} C_{\mathrm{IS}}}}{\sum \frac{\mathrm{AUC}}{\mathrm{AUC}} \mathrm{IS}}\right) \times 10
$$

Volcano plot was built by plotting, for each identified analyte, on $x$ axis, the log 2 of the fold change between $\left(\mathrm{AUC}_{\mathrm{n}}\right) /\left(\mathrm{AUC}_{\mathrm{IS}}\right) \mathrm{BLPF}$ vs. $\left(\mathrm{AUC}_{\mathrm{n}}\right) /\left(\mathrm{AUC}_{\mathrm{IS}}\right) \mathrm{BFPF}$ and on the $y$ axis the $-\log p$ value of the mean ratios. Those metabolites having a $\log 2$ fold change $\geq 1$ or $\leq-1$ and $-\log p$ value $\geq 2.5$ were considered to have a relative content significantly different in the two extracts.

\subsection{Quantitative Analysis of Selected Bergamot Components}

The major flavonoids (neoeriocitrin, naringin, neohesperidin, melitidin, and brutieridin) present in bergamot extracts were determined by chromatographic analysis with an HPLC system equipped with a DAD detector. Since the HPLC method was calibrated using naringin as standard, their concentration is expressed as naringin equivalent $(\mathrm{mg} / \mathrm{g}$ extract). $60 \mathrm{mg}$ of sample were dissolved in $20 \mathrm{~g}$ of a mixture of water-ethanol (50/50, $\% w / w)$. The resulting solution was heated to $50{ }^{\circ} \mathrm{C}$ and vortexed for complete dissolution. Prior to being introduced into the autosampler vial, the solution was filtered with a $0.2 \mu \mathrm{m}$ PTFE filter. The HPLC system used for the determination of the above flavonoids consists of a PerkinElmer Flexar Module equipped with a photodiode-array (PDA) detector, a series 200 autosampler, a series 200 peltier LC column oven, a series 200 LC pump, and a C18 Kinetex (particle size $5 \mu \mathrm{m}$, pore size $100 \mathrm{~A}$, length and diameter $150 \times 4.6 \mathrm{~mm}$ ) column (Phenomenex, Torrance, CA, USA). Control of the HPLC system and data collection was accomplished on-line by a computer equipped with Chromera ${ }^{\circledR}$ software (version 3.4.0.5712). Tests were performed in gradient mode with acetonitrile and water acidified by $0.1 \%$ of acetic acid $(88 \%, v / v)$ as eluents using a flow rate of $1.4 \mathrm{~mL} / \mathrm{min}$, an injection volume of $3.0 \mu \mathrm{L}$, and a wavelength of $284 \mathrm{~nm}$. The method was fully validated in terms of specificity, linearity, precision, accuracy, and quantitation limit (results are reported in the paragraph "Method S1. Method validation" of the Supplementary Materials).

\subsection{Evaluation of Proanthocyanidin Presence in the Extracts}

Proanthocyanidin presence was evaluated following two different methods. The first consisted of extract fractionation by gel permeation chromatography (GPC) according to the method developed by Gabetta et al. [26], with some modifications. GPC separations were performed at room temperature on a GE Healthcare Sephadex LH-20 (18-11 $\mu \mathrm{m}$ 
dry) column adapted for a Biotage Isolera ${ }^{\circledR}$ flash chromatography system. Sephadex LH$20(5.5 \mathrm{~g})$ which had previously been suspended in ethanol/acetone $(90 / 10, \% v / v)$ was then packed into a column $(7.0 \times 2.0 \mathrm{~cm}$ i.d). $200 \mathrm{mg}$ of each extract was dissolved in the initial mobile phases and loaded onto the pre-packed column. The separations were obtained by means of a binary linear gradient. The elution, under pressure, started using $10 \%$ of acetone and finished at $90 \%$ with $1000 \mathrm{~mL}$ as total volume of mobile phase at a constant flow rate of $5 \mathrm{~mL} / \mathrm{min}$. An aliquot of each of the obtained fractions was properly diluted in $\mathrm{MeOH} / \mathrm{HCOOH} \mathrm{100/0.1} \mathrm{( \% v/v)} \mathrm{and} \mathrm{analyzed} \mathrm{by} \mathrm{ESI-MS} \mathrm{(TSQ} \mathrm{Quantum} \mathrm{Ultra}$ Triple Quadrupole, Thermo Finnigan, Milan, Italy) in negative ion mode. The ion source parameters were set as following: spray voltage $-4.5 \mathrm{kV}$, sheath gas (nitrogen) 10 a.u., capillary temperature $275^{\circ} \mathrm{C}$, tube lens voltage $120 \mathrm{~V}$. As reference, Leucoselect ${ }^{\mathrm{TM}}$ grape seed selected proanthocyanidins. from $V$. vinifera seeds was also fractionated and analyzed.

The second approach is based on the concept that tannins (such as proanthocyanidins) are able to precipitate proteins rich in proline residues (PRPs). The tannin effect was evaluated for bergamot extracts, Leucoselect ${ }^{\mathrm{TM}}$ and tannic acid as references by applying the method developed by Baron et al. [27]. Each compound/extract was dissolved in $\mathrm{MeOH}$ as stock solution and diluted properly in order to be added 1:10 in the final mixture with bradykinin $(100 \mu \mathrm{M})$ in acetate buffer $50 \mathrm{mM}$. The mixtures were incubated for $10 \mathrm{~min}$ at $37^{\circ} \mathrm{C}$ with different concentrations of tannic acid $(0-200 \mu \mathrm{M})$, Leucoselect ${ }^{\mathrm{TM}}(0-2 \mathrm{mg} / \mathrm{mL})$, BFPF $(0-2 \mathrm{mg} / \mathrm{mL})$, and BLPF $(0-2 \mathrm{mg} / \mathrm{mL})$, respectively. Samples were then centrifuged, and an aliquot of the supernatant diluted in $\mathrm{H}_{2} \mathrm{O} / \mathrm{CH}_{3} \mathrm{CN} / \mathrm{HCOOH} 70 / 30 / 0.1(\% v / v)$, added with the peptide LVNEVTEF (used as internal standard) and analyzed by ESI-MS (TSQ Quantum Ultra Triple Quadrupole, Thermo Finnigan, Milan, Italy).

\subsection{Determination of Total Phenolic Content}

The total phenolic content was measured by a modified Folin-Ciocalteu colorimetric method [28]. The extracts were prepared at a concentration of $100 \mu \mathrm{g} / \mathrm{mL}$ to obtain absorbance values within the linearity range of the standard curve (gallic acid $0.0-100.0 \mu \mathrm{g} / \mathrm{mL}$ ). Aliquots of $100 \mu \mathrm{L}$ of the Folin-Ciocalteu reagent (FCR) and $1 \mathrm{~mL}$ of distilled water were added to $200 \mu \mathrm{L}$ of the extract. After 5 min standing, $700 \mu \mathrm{L}$ of a $7 \%$ sodium carbonate aqueous solution was added to the samples. After $90 \mathrm{~min}$ at room temperature, absorbance was measured at $760 \mathrm{~nm}$ in a 96 well plate using a plate reader (BioTek's PowerWave HT, Winooski, VT, USA). The total polyphenol content was expressed as $\mathrm{mg}$ of gallic acid equivalent per one gram of dry extract and reported as mean $\pm \mathrm{SD}$ for five replicates.

\subsection{Oxygen Radical Absorbing Capacity (ORAC) Assay}

The antioxidant capacity of the extracts against oxygen radicals was tested with the ORAC assay following the protocol of Wang et al. with some minor modifications [29]. The extracts were prepared in water/ethanol $(50 / 50, \% v / v)$ at different concentrations $(5-25 \mu \mathrm{g} / \mathrm{mL}$ ) and $20 \mu \mathrm{M}$ of trolox solution dissolved in the same solvents was used as reference. Aliquots of $250 \mu \mathrm{L}$ of sample were mixed with $250 \mu \mathrm{L}$ of a $2^{\prime}, 7^{\prime}$-dichloro-fluoresceine solution (500 nM) and $2 \mathrm{~mL}$ phosphate buffer $(75 \mathrm{mM}, \mathrm{pH} 7.0)$. After radical activation at $37^{\circ} \mathrm{C}$ for $10 \mathrm{~min}, 25 \mu \mathrm{L}$ of $2,2^{\prime}$-azobis(2-methylpropionamidine) dihydrochloride (ABAP) solution $(220 \mathrm{mM}$ ) was added to $475 \mu \mathrm{L}$ of sample. Fluorescence was measured using an excitation wavelength of $485 \mathrm{~nm}$ and emission at $535 \mathrm{~nm}$ (Wallac Victor2 1420, PerkinElmer ${ }^{\mathrm{TM}}$ Life Science, Monza, Italy). The antioxidant activity was calculated by measuring the differences of AUC between the extracts and the blank, and the results expressed as micromoles of trolox equivalents per one gram of extract. Values are reported as mean $\pm \mathrm{SD}$ of three replicates.

\subsection{ABTS Radical Cation Decolorization Assay}

The antioxidant activity against radical cation was also measured with the ABTS radical cation decolorization assay as reported by Re R. et al. [30]. ABTS radical cation (ABTS ${ }^{\bullet}$ ) was produced by reacting ABTS stock solution ( $7 \mathrm{mM}$ in water) with $2.45 \mathrm{mM}$ 
potassium persulfate (final concentration) and allowing the mixture to stand in the dark at room temperature for $12-16 \mathrm{~h}$ before use. The solution was then diluted in ethanol to an absorbance of $0.70( \pm 0.02)$ at $734 \mathrm{~nm}$. $180 \mu \mathrm{L}$ of the solution so obtained was added to $20 \mu \mathrm{L}$ of each sample analyzed in triplicate in a 96 well plate. A blank sample was also added to the plate. After $3 \mathrm{~min}$ at $30^{\circ} \mathrm{C}$ the absorbance was measured at $734 \mathrm{~nm}$ using a plate reader (BioTek's PowerWave HT, Winooski, VT, USA). The percentage of inhibition was calculated as expressed by Equation (2) and the results expressed as mean $\pm \mathrm{SD}$.

$$
\frac{\mathrm{Abs}_{\text {blank }}-\mathrm{Abs}_{\text {sample }}}{\mathrm{Abs}_{\text {blank }}} \times 100
$$

\subsection{DPPH (2,2-Diphenyl-1-Picrylhydrazyl) Assay}

The antioxidant capacity was also determined by the DPPH radical-scavenging method [31], with some modifications. An aliquot of $100 \mu \mathrm{L}$ of the extract solution at different concentrations $(1-25 \mu \mathrm{g} / \mathrm{mL})$ was added to $750 \mu \mathrm{L}$ of ethanol and $400 \mu \mathrm{L}$ of acetate buffer (100 mM, pH 5.5), mixed and spiked with $250 \mu \mathrm{L}$ of DPPH ethanolic solution $(500 \mu \mathrm{M})$. After $90 \mathrm{~min}$ at room temperature and in the dark the absorbance at $515 \mathrm{~nm}$ was measured for each sample analyzed in triplicate with a UV reader Shimadzu ${ }^{\mathrm{TM}}$ UV 1900 (Shimadzu, Milano, Italia). The percentage of inhibition was calculated as expressed by Equation (3) and the results expressed as mean \pm SD.

$$
\frac{\mathrm{Abs}_{\text {blank }}-\mathrm{Abs}_{\text {sample }}}{\mathrm{Abs}_{\text {blank }}} \times 100
$$

\subsection{Cell Culture and Cell Stimulation}

HEK293T (ATCC ${ }^{\circledR}$, Manassas, VA, USA; accession number: CRL-3216 ${ }^{\mathrm{TM}}$ ) and rat alveolar type I-like R3/1 clones were grown in Dulbecco modified Eagle medium (DMEM; Lonza, Verviers, Belgium) supplemented with 10\% fetal bovine serum (FBS; Gibco, Gaithersburg, MD, USA), 1\% glutamine (Lonza), and 1\% penicillin/streptomycin (Gibco, Gaithersburg, MD, USA). R3/1-pLXSN cells (R3/1 control [32]) were used to generate, by lentiviral infection, a stable NF- $\kappa B$ signaling pathway reporter cell line (R3/1-NF- $\kappa B$ ) using the lentivector $\mathrm{pGreenFire-NF- \kappa B-Puro} \mathrm{(a} \mathrm{kind} \mathrm{gift} \mathrm{from} \mathrm{Dr.} \mathrm{Darius} \mathrm{Widera,} \mathrm{University} \mathrm{of}$ Reading, UK; [33]) which drives the expression of both red firefly luciferase reporter and GFP in response to NF- $\mathrm{B}$ activity.

For lentivirus production, $9 \times 10^{6} 293 \mathrm{~T}$ cells were seeded in a $15 \mathrm{~cm}$ plate and after $16 \mathrm{~h}$ the medium was changed to Iscove's Modified Dulbecco's Medium (IMDM; Lonza) containing 10\% FBS, 1\% penicillin/streptomycin and 1\% glutamine $100 \mathrm{U} / \mathrm{mL}$. Calcium phosphate precipitation method was used for transfection of lentiviral package vectors (7 $\mu \mathrm{g}$ pDM2-VSVG and $28 \mu \mathrm{g}$ pCMV- $\Delta$ R8.91) and $32 \mu \mathrm{g}$ pGreenFire-NF- $\mathrm{BB}-\mathrm{Puro}$. The medium was changed after $16 \mathrm{~h}$ to complete IMDM and butyrate sodium $(1 \mu \mathrm{g} / \mathrm{mL}$; Sigma, St Louis, MO, USA) and medium containing virus particles was collected $36 \mathrm{~h}$ later, centrifuged at $1500 \mathrm{rpm}$ for $5 \mathrm{~min}$, filtered through $0.22 \mu \mathrm{m}$ filter (Merck, Germany) and centrifuged again at 20,000 rpm for $2 \mathrm{~h}$ at $4{ }^{\circ} \mathrm{C}$. Viral particles were dissolved in $40 \mu \mathrm{L}$ of sterile cold PBS and left in ice with moderate agitation for $30 \mathrm{~min}$. Finally, virus aliquots were kept at $-80^{\circ} \mathrm{C}$. For lentiviral transduction, R3/1 control cells $(60,000)$ were seeded in a 12 well-plate and the next day fresh medium containing $6 \mu \mathrm{L}$ (MOI 10) of virus particles was added for $24 \mathrm{~h}$. Cells were then selected by treating cells with $1 \mu \mathrm{g} / \mathrm{mL}$ of puromycin for 4 days. Selected R3/1-NF-кB -cells were seeded at 3000 cells / well in a 96-wells plate for subsequent experiments.

To assess the anti-inflammatory activity, R3/1-NF- $\mathrm{BB}$ cells were pre-treated with the two extracts at different concentrations $(10-250 \mu \mathrm{g} / \mathrm{mL})$ for $18 \mathrm{~h}$ in complete medium, followed by a $6-\mathrm{h}$ stimulation with $10 \mathrm{ng} / \mathrm{mL}$ IL- $1 \alpha$. In order to verify a possible direct interaction between the extract and IL- $1 \alpha$, the addition was performed with and without the removal of the medium containing the extract. Experiments were assayed by NF- $k \mathrm{~B}$ luciferase activity, as described below. 


\subsection{NF-kB Luciferase Activity Assay}

After treatment, R3/1-NF-kBcells were washed twice with cold PBS followed by a freeze-thaw cycle with reporter lysis buffer (purchased from Promega Corporation, Madison, WI, USA) for complete cell lysis. After the freeze-thaw cycle, $100 \mu \mathrm{L}$ ONE-Glo ${ }^{\mathrm{TM}}$ Luciferase Assay Substrate (purchased from Promega Corporation, Madison, WI, USA) was directly added to the wells, followed by a luciferase measurement performed using a luminometer (Wallac Victor2 1420, Perkin-Elmer ${ }^{\mathrm{TM}}$ Life Science, Monza, Italy).

\subsection{MTT Assay}

The cell viability for the all the concentrations tested in the anti-inflammatory assay was verified by MTT assay on R3/1-NF- $\mathrm{kB}$ cells. After $18 \mathrm{~h}$ incubation with rosiglitazone $(1-75 \mu \mathrm{M})$ and the extracts $(10-250 \mu \mathrm{g} / \mathrm{mL}), 10 \mu \mathrm{L} 5 \mathrm{mg} / \mathrm{mL}$ MTT reagent was added for $4 \mathrm{~h}$. After medium removal, R3/1-NF- $\mathrm{kB}$ cells were lysed and MTT was solubilized by adding $100 \mu \mathrm{L}$ of DMSO. The 96-well plate was shaken for $1 \mathrm{~min}$ and the absorbance at $490 \mathrm{~nm}$ was measured using a plate reader (BioTek's PowerWave HT, Winooski, VT, USA). Cells incubated with DMSO $(<0.1 \%)$ were used as a control for $100 \%$ cell proliferation.

\subsection{Statistical Analysis}

Biological experiments were performed with biological and technical replicates. Values are shown as mean \pm SEM compared to untreated control cells. Statistical analysis was performed by using one-way ANOVA with Bonferroni correction. $p<0.05$ was considered significant. Statistical analysis was performed with GraphPad Prism 6.02 for Windows, GraphPad Software, La Jolla, CA, USA, (www.graphpad.com) and OriginPro, version 2019, OriginalLab Corporation, Northampton, MA, USA.

\section{Results}

\subsection{Targeted, Semi-Targeted and Untargeted Profiling by LC-HRMS}

The analytical qualitative profile of the two extracts was carried out by using three different approaches: targeted, semi-targeted and untargeted. The first consisted of building a database of bergamot components already reported in the literature and containing 89 entries (Table S1 of Supplementary Materials). The identification of extract components in the $\mathrm{db}$ was made by matching the accurate masses, isotopic and fragmentation patterns. When possible, the identification of stereoisomers was performed on the basis of the elution order reported in previous papers which used RP chromatography as separation technique. The second approach (semi-targeted) was aimed at selectively identifying the most intense HMG derivatives which have interesting biological effects since they are involved in the cholesterol reducing activity of BPF; the approach consisted of mapping the characteristic ion losses of the HMG moiety (-62 Da, -102 Da and -144 Da): three different ion maps were generated from each of these losses and the common precursors were manually verified, thus giving a list of HMG-containing compounds. The putative identification was then accomplished by the accurate mass, isotopic and fragmentation patterns and by considering the match with molecular formula. The untargeted approach was focused on the identification of the most intense ions not identified using the previous methods: the elemental composition was calculated by the QualBrowser tool of Xcalibur as reported in the method section; the molecular formula was searched for using online databases such as MoNa and HMDB which generated a list of possible candidates whose structures had undergone in silico fragmentations. Simulated fragment ions were then compared to the experimental ones through the Peak Assignment tool of CFM-ID online software and the compounds putatively identified on the basis of the best fragmentation match $[34,35]$. A total number of 108 compounds were identified by using targeted, semitargeted and untargeted profiling in bergamot leaf and fruit extracts, 100 of which are present in both the extracts thus demonstrating a good overlapping, at least from a qualitative point of view. Table 1 reports the retention times, accurate masses and fragmentation patterns of the metabolites identified by using the targeted approach; of the 61 metabo- 
lites identified, six were organic and phenolic acids, 24 flavones, 24 flavanones and seven limonoids. Most were present in both extracts, except for apigenin-7-O-rutinoside and neohesperidin-O-glucoside- $\mathrm{O}-\mathrm{HMG}$ which were detected only in the leaf extract, while obacunone glucoside, limonin glucoside and obacunoic acid were detected only in BFPF. Some isomers were identified on the basis of their elution order as reported in previous studies which used RP chromatography as separation technique: for chrysoeriol/diosmetin isomers, chrysoeriol has always been reported as the first eluting isomer [18,19,21,25]; similarly, for rutinoside/neohesperidoside isomers the rutinoside is always the first eluting isomer followed by neohesperidoside $[9,15-19,21-23,25]$. Figure 1 (panels a and b) shows the total ion currents (TICs) of the two extracts where the peak ions identified by a targeted approach are labelled in blue by a time-dependent progressive number.

Table 1. Compounds identified in both BFPF and BLPF with the targeted approach. * only present in the BLPF. ${ }^{* *}$ only present in BFPF.

\begin{tabular}{|c|c|c|c|c|c|c|}
\hline Peak & Compound & RT (min) & $m / z$ & MS/MS Ion Fragments & $\begin{array}{l}\text { Molecular } \\
\text { Formula }\end{array}$ & \\
\hline \multicolumn{7}{|c|}{ Non-phenolics } \\
\hline \multicolumn{7}{|c|}{ Organic Acids } \\
\hline 1 & Citric acid & 2.5 & 191.0202 & $111-147$ & $\mathrm{C}_{6} \mathrm{H}_{8} \mathrm{O}_{7}$ & 1.571 \\
\hline \multicolumn{7}{|c|}{ Limonoids } \\
\hline 28 & Nomilin glucoside & 41.8 & 693.2748 & $427-471-565-607-633-651$ & $\mathrm{C}_{34} \mathrm{H}_{46} \mathrm{O}_{15}$ & -0.517 \\
\hline 30 & Nomilinic acid glucoside & 45.0 & 711.2850 & $607-651$ & $\mathrm{C}_{34} \mathrm{H}_{48} \mathrm{O}_{16}$ & -0.862 \\
\hline 33 & Obacunone glucoside ${ }^{* *}$ & 48.9 & 633.2535 & 331-359-427-589 & $\mathrm{C}_{32} \mathrm{H}_{42} \mathrm{O}_{13}$ & -0.668 \\
\hline 35 & Limonin glucoside ** & 49.8 & 649.2502 & 341-385-443-461-587-605 & $\mathrm{C}_{32} \mathrm{H}_{42} \mathrm{O}_{14}$ & 1.118 \\
\hline 40 & Obacunoic acid ${ }^{* *}$ & 58.8 & 471.2017 & $203-245-307-325-351-409-427$ & $\mathrm{C}_{26} \mathrm{H}_{32} \mathrm{O}_{8}$ & 0.346 \\
\hline 41 & Limonin & 60.1 & 469.1872 & 229-278-283-306-321-381 & $\mathrm{C}_{26} \mathrm{H}_{30} \mathrm{O}_{8}$ & 1.506 \\
\hline 42 & Nomilinic acid & 60.6 & 531.2220 & 427-471-489 & $\mathrm{C}_{28} \mathrm{H}_{36} \mathrm{O}_{10}$ & -0.474 \\
\hline \multicolumn{7}{|c|}{ Phenols } \\
\hline \multicolumn{7}{|c|}{ Phenolic Acids } \\
\hline 2 & Feruloyl glucoside isomer 1 & 5.2 & 355.1035 & 193 & $\mathrm{C}_{16} \mathrm{H}_{20} \mathrm{O}_{9}$ & 3.271 \\
\hline 3 & Sinapoyl glucoside isomer 1 & 6.4 & 385.1135 & 223 & $\mathrm{C}_{17} \mathrm{H}_{22} \mathrm{O}_{10}$ & 0.567 \\
\hline 5 & Feruloyl glucoside isomer 2 & 8.0 & 355.1033 & 193 & $\mathrm{C}_{16} \mathrm{H}_{20} \mathrm{O}_{9}$ & 0.941 \\
\hline 6 & $\begin{array}{c}\text { Sinapoyl glucoside isomer } 2 \\
\text { 2-Hydroxy-4- }\end{array}$ & 8.6 & 385.1136 & 223 & $\mathrm{C}_{17} \mathrm{H}_{22} \mathrm{O}_{10}$ & 0.697 \\
\hline 9 & $\begin{array}{c}\text { methoxyhydrocinnamoyl-2- } \\
\text { O-glucoside }\end{array}$ & 13.0 & 357.1185 & 151-177-195 & $\mathrm{C}_{16} \mathrm{H}_{22} \mathrm{O}_{9}$ & 0.491 \\
\hline \multicolumn{7}{|c|}{ Polyphenols } \\
\hline \multicolumn{7}{|c|}{ Flavones } \\
\hline 4 & Luteolin-6,8-di-C-glucoside & 7.2 & 609.1443 & $368-399-429-471-489-519$ & $\mathrm{C}_{27} \mathrm{H}_{30} \mathrm{O}_{16}$ & -0.711 \\
\hline 7 & Apigenin-6,8-di-C-glucoside & 10.2 & 593.1495 & 353-383-473-503 & $\mathrm{C}_{27} \mathrm{H}_{30} \mathrm{O}_{15}$ & -0.596 \\
\hline 8 & $\begin{array}{l}\text { Chrysoeriol-6,8-di-C- } \\
\text { glucoside }\end{array}$ & 12.1 & 623.1600 & $312-383-413-503-533$ & $\mathrm{C}_{28} \mathrm{H}_{32} \mathrm{O}_{16}$ & -0.661 \\
\hline 10 & Diosmetin-6,8-di-C-glucoside & 13.7 & 623.1603 & $312-383-413-503-533$ & $\mathrm{C}_{28} \mathrm{H}_{32} \mathrm{O}_{16}$ & -0.361 \\
\hline 11 & Luteolin-7-O-glucoside & 15.1 & 447.0924 & 285 & $\mathrm{C}_{21} \mathrm{H}_{20} \mathrm{O}_{11}$ & 0.212 \\
\hline 13 & Apigenin-8-C-glucoside & 20.7 & 431.0983 & 269-283-311-341 & $\mathrm{C}_{21} \mathrm{H}_{20} \mathrm{O}_{10}$ & 1.027 \\
\hline \multirow[t]{2}{*}{14} & Apigenin-6-C-glucoside & 21.8 & 431.0979 & 269-283-311-341 & $\mathrm{C}_{21} \mathrm{H}_{20} \mathrm{O}_{10}$ & 0.627 \\
\hline & Chrysoeriol-8-C-glucoside & 25.9 & 461.1084 & 341-371 & $\mathrm{C}_{22} \mathrm{H}_{22} \mathrm{O}_{11}$ & 0.562 \\
\hline 16 & $\begin{array}{l}\text { Luteolin-7-O- } \\
\text { neohesperidoside }\end{array}$ & 26.5 & 593.1496 & $285-447$ & $\mathrm{C}_{27} \mathrm{H}_{30} \mathrm{O}_{15}$ & -0.496 \\
\hline 17 & Diosmetin-8-C-glucoside & 27.0 & 461.1090 & $341-371$ & $\mathrm{C}_{22} \mathrm{H}_{22} \mathrm{O}_{11}$ & 1.162 \\
\hline 20 & Apigenin-7-O-rutinoside * & 30.4 & 577.1575 & 269 & $\mathrm{C}_{27} \mathrm{H}_{30} \mathrm{O}_{14}$ & 2.318 \\
\hline 21 & $\begin{array}{l}\text { Apigenin-7-O- } \\
\text { neohesperidoside }\end{array}$ & 33.2 & 577.1557 & 269 & $\mathrm{C}_{27} \mathrm{H}_{30} \mathrm{O}_{14}$ & 0.518 \\
\hline 23 & Chrysoeriol-7-O-glucoside & 35.0 & 461.1079 & 284-299 & $\mathrm{C}_{22} \mathrm{H}_{22} \mathrm{O}_{11}$ & 0.062 \\
\hline 24 & Diosmetin-7-O-glucoside & 35.8 & 461.1082 & $284-299$ & $\mathrm{C}_{22} \mathrm{H}_{22} \mathrm{O}_{11}$ & 0.362 \\
\hline 25 & $\begin{array}{l}\text { Chrysoeriol-7-O- } \\
\text { neohesperidoside }\end{array}$ & 36.2 & 607.1655 & 284-299 & $\mathrm{C}_{28} \mathrm{H}_{32} \mathrm{O}_{15}$ & -0.247 \\
\hline 25 & $\begin{array}{l}\text { Demethoxycentaureidin-7-O- } \\
\text { glucoside }\end{array}$ & 36.3 & 491.1199 & 314-329-371 & $\mathrm{C}_{23} \mathrm{H}_{24} \mathrm{O}_{12}$ & 1.498 \\
\hline 27 & $\begin{array}{c}\text { Diosmetin-7-O- } \\
\text { neohesperidoside }\end{array}$ & 37.9 & 607.1655 & 284-299 & $\mathrm{C}_{28} \mathrm{H}_{32} \mathrm{O}_{15}$ & -0.247 \\
\hline 32 & $\begin{array}{c}\text { Apigenin-7-O- } \\
\text { neohesperidoside-O-HMG }\end{array}$ & 47.2 & 721.1959 & 577-619-659 & $\mathrm{C}_{33} \mathrm{H}_{38} \mathrm{O}_{18}$ & -1.541 \\
\hline
\end{tabular}


Table 1. Cont.

\begin{tabular}{|c|c|c|c|c|c|c|}
\hline Peak & Compound & RT (min) & $m / z$ & MS/MS Ion Fragments & $\begin{array}{l}\text { Molecular } \\
\text { Formula }\end{array}$ & \\
\hline 34 & Luteolin & 49.1 & 285.0400 & 151-175-191-199-217-241-243 & $\mathrm{C}_{15} \mathrm{H}_{10} \mathrm{O}_{6}$ & 0.636 \\
\hline 34 & $\begin{array}{c}\text { Diosmetin-7-O- } \\
\text { neohesperidoside-O-HMG }\end{array}$ & 49.1 & 751.2095 & 299-461-607-649-689 & $\mathrm{C}_{34} \mathrm{H}_{40} \mathrm{O}_{19}$ & 1.495 \\
\hline 36 & $\begin{array}{l}\text { Demethoxycentaureidin-7-O- } \\
\text { glucoside-HMG }\end{array}$ & 50.9 & 635.1604 & $314-329-491-533$ & $\mathrm{C}_{29} \mathrm{H}_{32} \mathrm{O}_{16}$ & -0.261 \\
\hline 38 & Apigenin & 54.5 & 269.0450 & $149-175-225$ & $\mathrm{C}_{15} \mathrm{H}_{10} \mathrm{O}_{5}$ & 0.550 \\
\hline 39 & Chrysoeriol & 55.5 & 299.0557 & 256-271-284 & $\mathrm{C}_{16} \mathrm{H}_{12} \mathrm{O}_{6}$ & 0.685 \\
\hline \multirow[t]{3}{*}{39} & Diosmetin & 55.7 & 299.0558 & $256-284$ & $\mathrm{C}_{16} \mathrm{H}_{12} \mathrm{O}_{6}$ & 0.785 \\
\hline & Flavanones & & & & & \\
\hline & Naringin-glucoside & 14.7 & 741.2249 & $271-459-479$ & $\mathrm{C}_{33} \mathrm{H}_{42} \mathrm{O}_{19}$ & 1.245 \\
\hline 12 & $\begin{array}{l}\text { Eriodictyol 7-O-rutinoside } \\
\text { (Eriocitrin) }\end{array}$ & 20.0 & 595.1657 & 287 & $\mathrm{C}_{27} \mathrm{H}_{32} \mathrm{O}_{15}$ & -0.047 \\
\hline 13 & $\begin{array}{l}\text { Eriodictyol-7-O-glucoside } \\
\text { Eriodictyol }\end{array}$ & 20.5 & 449.1080 & 287 & $\mathrm{C}_{21} \mathrm{H}_{22} \mathrm{O}_{11}$ & 0.162 \\
\hline 15 & $\begin{array}{l}\text { 7-O-neohesperidoside } \\
\text { (Neoeriocitrin) }\end{array}$ & 22.9 & 595.1651 & $287-449$ & $\mathrm{C}_{27} \mathrm{H}_{32} \mathrm{O}_{15}$ & -0.647 \\
\hline 18 & $\begin{array}{l}\text { Naringenin 7-O-rutinoside } \\
\text { (Narirutin) }\end{array}$ & 27.6 & 579.1713 & 271 & $\mathrm{C}_{27} \mathrm{H}_{32} \mathrm{O}_{14}$ & 0.468 \\
\hline 18 & $\begin{array}{c}\text { Neoeriocitrin-glucoside-O- } \\
\text { HMG }\end{array}$ & 28.0 & 901.2591 & $\begin{array}{c}\text { 287-595-637-377-739-757-799- } \\
839\end{array}$ & $\mathrm{C}_{39} \mathrm{H}_{50} \mathrm{O}_{24}$ & -1.728 \\
\hline 19 & $\begin{array}{l}\text { Naringenin-7-O-glucoside } \\
\text { (Prunasin) }\end{array}$ & 29.4 & 433.1133 & 271 & $\mathrm{C}_{21} \mathrm{H}_{22} \mathrm{O}_{10}$ & 0.377 \\
\hline 19 & $\begin{array}{c}\text { Bergamjuicin } \\
\text { (Melitidin-glucoside) }\end{array}$ & 29.5 & 885.2640 & $\begin{array}{c}271-459-579-621-661-723-741- \\
783-823\end{array}$ & $\mathrm{C}_{39} \mathrm{H}_{50} \mathrm{O}_{23}$ & -1.914 \\
\hline 20 & $\begin{array}{c}\text { Neohesperidin-glucoside- } O \text { - } \\
\text { HMG } \\
*\end{array}$ & 30.3 & 915.2730 & $301-609-651-691-771-813-853$ & $\mathrm{C}_{40} \mathrm{H}_{52} \mathrm{O}_{24}$ & -3.479 \\
\hline \multirow[t]{2}{*}{20} & $\begin{array}{l}\text { Naringenin } \\
\text { 7-O-neohesperidoside } \\
\text { (Naringin) }\end{array}$ & 30.5 & 579.1705 & 271 & $\mathrm{C}_{27} \mathrm{H}_{32} \mathrm{O}_{14}$ & -0.332 \\
\hline & $\begin{array}{l}\text { Hesperetin- } O \text {-glucoside } \\
\text { isomer } 1\end{array}$ & 32.4 & 463.1241 & 301 & $\mathrm{C}_{22} \mathrm{H}_{24} \mathrm{O}_{11}$ & 0.612 \\
\hline 22 & $\begin{array}{l}\text { Hesperetin 7-O-rutinoside } \\
\text { (Hesperidin) }\end{array}$ & 33.6 & 609.1816 & $301-489$ & $\mathrm{C}_{28} \mathrm{H}_{34} \mathrm{O}_{15}$ & 0.203 \\
\hline 25 & $\begin{array}{c}\text { Hesperetin- } O \text {-glucoside } \\
\text { isomer } 2 \\
\text { Hesperetin }\end{array}$ & 36.3 & 463.1239 & 301 & $\mathrm{C}_{22} \mathrm{H}_{24} \mathrm{O}_{11}$ & 0.412 \\
\hline 26 & $\begin{array}{l}\text { 7-O-neohesperidoside } \\
\text { (Neohesperidin) }\end{array}$ & 36.7 & 609.1809 & $301-447-489$ & $\mathrm{C}_{28} \mathrm{H}_{34} \mathrm{O}_{15}$ & -0.497 \\
\hline 27 & Neoeriocitrin-O-HMG & 37.6 & 739.2067 & 287-433-595-637-677 & $\mathrm{C}_{33} \mathrm{H}_{40} \mathrm{O}_{19}$ & -1.305 \\
\hline 29 & Eriodictyol & 42.6 & 287.0560 & $135-151$ & $\mathrm{C}_{15} \mathrm{H}_{12} \mathrm{O}_{6}$ & 0.985 \\
\hline 30 & $\begin{array}{c}\text { Naringenin } \\
\text { 7-O-glucoside-O-HMG }\end{array}$ & 44.8 & 577.1554 & 271-433-475-515 & $\mathrm{C}_{27} \mathrm{H}_{30} \mathrm{O}_{14}$ & 0.378 \\
\hline 31 & $\begin{array}{l}\text { Melitidin (Naringin-O-HMG) } \\
\text { Hesperetin-O-glucoside-O- }\end{array}$ & 45.7 & 723.2148 & $579-621-661$ & $\mathrm{C}_{33} \mathrm{H}_{40} \mathrm{O}_{18}$ & 1.709 \\
\hline 32 & HMG isomer & 47.1 & 607.1666 & 301-463-505-545 & $\mathrm{C}_{28} \mathrm{H}_{32} \mathrm{O}_{15}$ & 0.853 \\
\hline 34 & $\begin{array}{c}\text { Hesperetin- } O \text {-glucoside- } O \text { - } \\
\text { HMG isomer } \\
2\end{array}$ & 49.8 & 607.1651 & $301-463-505-545$ & $\mathrm{C}_{28} \mathrm{H}_{32} \mathrm{O}_{15}$ & -0.647 \\
\hline 35 & $\begin{array}{c}\text { Brutieridin } \\
\text { (Neohesperidin-O-HMG) }\end{array}$ & 50.0 & 753.2223 & 609-651-691 & $\mathrm{C}_{34} \mathrm{H}_{42} \mathrm{O}_{19}$ & -1.355 \\
\hline 37 & Naringenin & 53.2 & 271.0608 & $107-119-151-165-177-227$ & $\mathrm{C}_{15} \mathrm{H}_{12} \mathrm{O}_{5}$ & 0.700 \\
\hline 38 & $\begin{array}{c}\text { Isosakuranetin-7-O- } \\
\text { neohesperidoside-O-HMG }\end{array}$ & 54.4 & 737.2266 & 285-411-593-635-675 & $\mathrm{C}_{34} \mathrm{H}_{42} \mathrm{O}_{18}$ & 2.596 \\
\hline 39 & Hesperetin & 55.3 & 301.0714 & $151-174-199-242-258-268$ & $\mathrm{C}_{16} \mathrm{H}_{14} \mathrm{O}_{6}$ & 0.735 \\
\hline
\end{tabular}



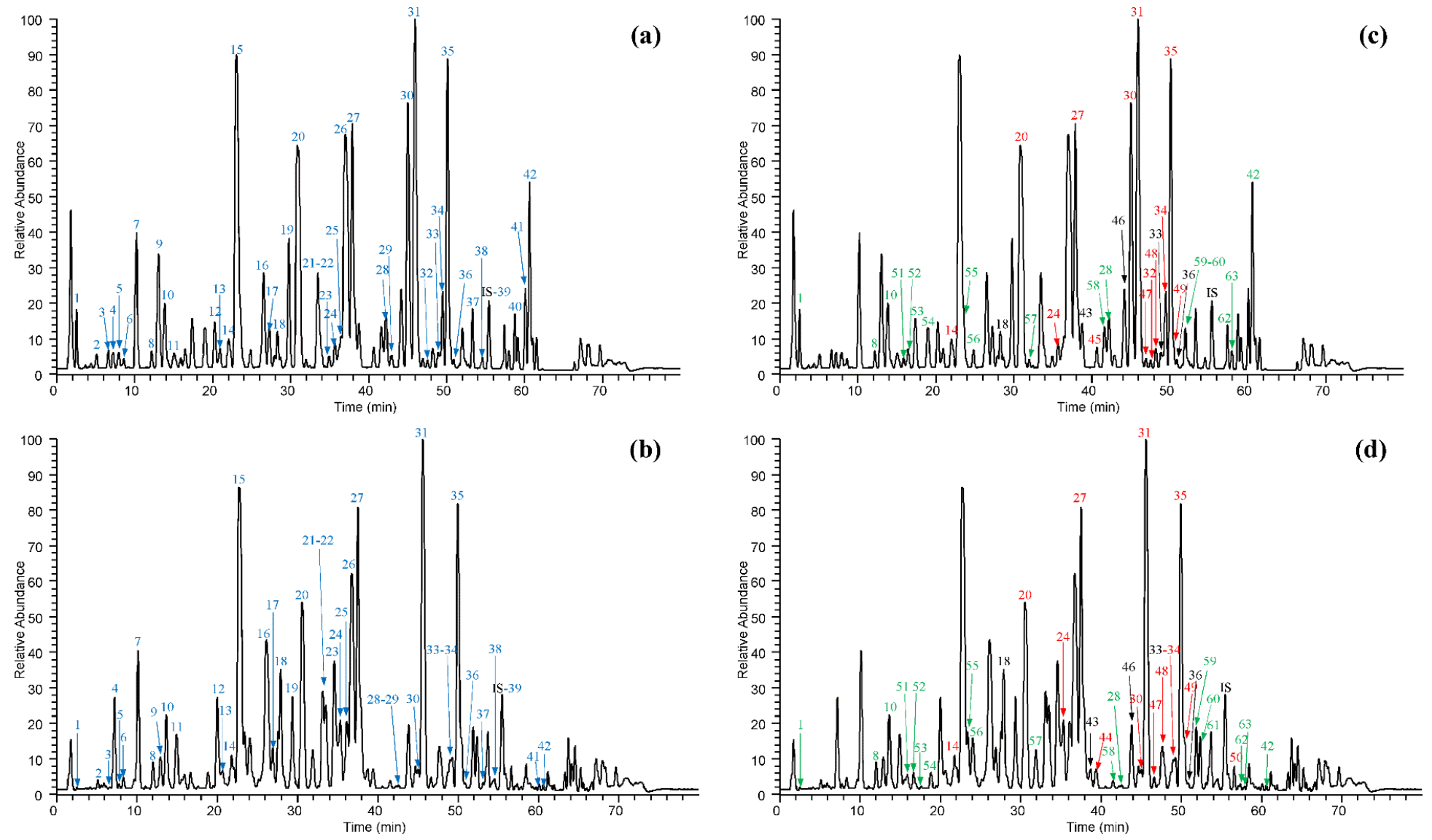

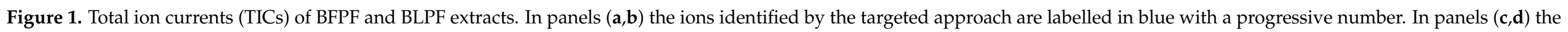
ions identified with the semi-targeted and untargeted approaches are labeled in red and green, respectively. (IS) Internal standard (Trolox); BFPF: panels (a,c); BLPF panels (b,d). 
Table 2 lists the 32 ions identified with the semi-targeted approach, including 11 compounds which were definitively confirmed since they had already been identified with the targeted approach. Of the remaining 21 compounds not yet identified in the bergamot plant, 17 were putatively assigned on the basis of the accurate mass, molecular formula, isotopic and fragmentation patterns while four HMG derivatives remain unknown. Thus, this approach allowed the detection of $20 \mathrm{HMG}$ derivatives hitherto unreported in the literature, 17 found in both extracts, two only in leaf extract (luteolin- $O$-glucoside- $O-H M G$ and an acetyl-glucosyl-O-HMG derivative), and one only in the fruit extract (bergamjuicin glucoside). Of these HMG derivatives, 6 out of 21 are flavone or flavanone di-glucosides, characterized by the loss of the HMG moiety ( $-144 \mathrm{Da}$ ) and by two neutral losses at $-162 \mathrm{Da}$ and $-120 \mathrm{Da}$ for the O-glucoside and C-glucoside, respectively; four are flavone mono glucosides, characterized by the neutral loss of the HMG moiety and of the sugar. The two flavanone rutinosides were recognized by the loss of the HMG moiety and by the loss of glucose and rhamnose $(-146 \mathrm{Da})$. Bergamjuicin glucoside was characterized by the presence of an additional glucose moiety on the bergamjuicin structure. The compound 6-(beta-D-glucopyranosyloxy)-4-methoxy-5-benzofuranpropanoic acid-O-HMG was tentatively assigned on the basis of the identification of the HMG moiety and of the 6-(beta-D-glucopyranosyloxy)-4-methoxy-5-benzofuranpropanoic acid residue, this last annotated through the similarity search spectra of the MoNa database in the untargeted approach. Four identified HMG compounds characterized by the aglycones at $\mathrm{m} / \mathrm{z} 315$ (two isomers), 255 and 201 were not assigned. Table 3 lists the 26 compounds putatively identified by the untargeted method. MS and MS/MS spectra used for the putative identifications are collected in Figures S1-S26 of Supplementary Materials. Quinic acid has been previously identified in bergamot as an ester of sinapic acid [23], but not in a free form as in the current work. HMG-glucoside is here identified for the first time in bergamot and could derive from the hydrolysis of compounds bearing this moiety, such as melitidin. The two compounds 6-(beta-D-glucopyranosyloxy)-5-benzofuranpropanoic acid (also known as cnidioside A) and 6-(beta-D-glucopyranosyloxy)-4-methoxy-5-benzofuranpropanoic acid (also known as picraquassioside A) had also never been reported in bergamot although both of them were identified in an ethanolic extract of Ruta graveolens [36,37], which belongs to the Rutaceae family as does bergamot. The ion at $m / z 265.1072$ (compound 59) was tentatively assigned as 3-[2,4,5-trihydroxy-3-(3-methylbut-2-en-1-yl) phenyl] propanoic acid on the basis of its similarity to (3-[3,4-dihydroxy-5-(3-methylbut-2-en-1-yl) phenyl]-2hydroxypropanoic acid), the latter was identified through the Compound identification tool of CFM-ID. The two compounds differ in the position of the hydroxy moiety, and the position on the assigned structure was made by considering that it can derive from compounds already present in bergamot and in particular by the opening of coumarin or by prenylation of the phenyl-propanoic acid moiety. Many of the compounds here putatively identified and not yet reported in the literature are glycosides of flavones and flavanones known to be present in bergamot, namely: neoeriocitrin- $O$-glucoside (or eriocitrin- $O$-glucoside), luteolin- $\mathrm{O}$-neohesperidoside- $\mathrm{O}$-glucoside (found in Citrus juices [38]), luteolin-C-glucoside$O$-rhamnoside, luteolin-O-rutinoside, luteolin- $O$-neohesperidoside- $O$-rhamnoside, hesperetindi-C-glucoside, naringenin-C-neohesperidoside- $O$-rhamnoside, apigenin- $O$-glucoside. Some other compounds are acetyl-derivatives of already known flavone and flavanone glycosides, such as luteolin- $\mathrm{O}$-acetyl-O-neohesperidoside, naringenin-C-glucoside- $\mathrm{O}$-acetylrhamnoside, diosmetin-O-acetyl-neohesperidoside (found in Citrus aurantium [39]). Figure 1 shows the TIC traces of BFPF and BLPF where peak ions identified by the semi-targeted and untargeted methods are labelled in red and green, respectively. Peaks reporting more than one number indicate the co-elution of more than one ion. For each identified compound, the mass spectrum together with the isotopic pattern and the relative MS/MS spectrum were analyzed and where possible, the fragmentation tree generated. As an example, Figure 2 shows the MS and MS/MS results relative to apigenin-di-O-glucoside. 
Table 2. Compounds identified * and putatively identified ${ }^{* *}$ with the semi-targeted method divided for flavonoid classes. In bold the compounds only present in BLPF, in italics those only present in BFPF.

\begin{tabular}{|c|c|c|c|c|c|c|}
\hline Peak & $\begin{array}{l}\text { Identification/Putative } \\
\text { Identification }\end{array}$ & RT (min) & $m / z$ & $\begin{array}{l}\text { MS/MS Ion } \\
\text { Fragments }\end{array}$ & $\begin{array}{l}\text { Molecular } \\
\text { Formula }\end{array}$ & $\Delta \mathrm{ppm}$ \\
\hline \multicolumn{7}{|c|}{ Polyphenols } \\
\hline \multirow{6}{*}{14} & Flavones & & & & & \\
\hline & $\begin{array}{c}\text { Apigenin-di-O-glucoside- } O- \\
\text { HMG }\end{array}$ & 21.4 & 737.1903 & $\begin{array}{l}269-431-593- \\
635-675\end{array}$ & $\mathrm{C}_{33} \mathrm{H}_{38} \mathrm{O}_{19}$ & -2.774 \\
\hline & $\begin{array}{c}\text { Diosmetin-di-C-glucoside- } O- \\
\mathrm{HMG}^{* *}\end{array}$ & 25.6 & 767.1998 & $\begin{array}{l}299-341-461- \\
\quad 605-647\end{array}$ & $\mathrm{C}_{34} \mathrm{H}_{40} \mathrm{O}_{20}$ & -4.066 \\
\hline & $\begin{array}{c}\text { Chrysoeriol-di-O-glucoside- } O- \\
\text { HMG }\end{array}$ & 28.6 & 767.2006 & 299-461-503-605 & $\mathrm{C}_{34} \mathrm{H}_{40} \mathrm{O}_{20}$ & -3.024 \\
\hline & $\begin{array}{c}\text { Diosmetin-di-O-glucoside- } O- \\
\mathrm{HMG}^{* *}\end{array}$ & 29.7 & 767.1998 & 299-461-503-605 & $\mathrm{C}_{34} \mathrm{H}_{40} \mathrm{O}_{20}$ & -4.066 \\
\hline & $\begin{array}{c}\text { Luteolin- } O \text {-glucoside- } O \text { - } \\
\text { HMG }\end{array}$ & 37.4 & 591.1353 & $285-447-489-529$ & $\mathrm{C}_{27} \mathrm{H}_{28} \mathrm{O}_{15}$ & 1.444 \\
\hline 44 & $\begin{array}{l}\text { Luteolin-O-neohesperidoside- } O \text { - } \\
\text { HMG }\end{array}$ & 39.5 & 737.1934 & $\begin{array}{l}285-447-593- \\
\quad 635-675\end{array}$ & $\mathrm{C}_{33} \mathrm{H}_{38} \mathrm{O}_{19}$ & 1.417 \\
\hline 47 & $\begin{array}{c}\text { Apigenin- } O \text {-glucoside- } O- \\
\mathrm{HMG}^{* *}\end{array}$ & 46.6 & 575.1403 & $269-431-473-513$ & $\mathrm{C}_{27} \mathrm{H}_{28} \mathrm{O}_{14}$ & 1.336 \\
\hline 32 & $\begin{array}{l}\text { Apigenin-7-O-neohesperidoside- } \\
\text { O-HMG }\end{array}$ & 47.2 & 721.1967 & $577-619-659$ & $\mathrm{C}_{33} \mathrm{H}_{38} \mathrm{O}_{18}$ & -1.027 \\
\hline 33 & $\begin{array}{c}\text { Chrysoeriol-O-glucoside- } O- \\
\text { HMG }\end{array}$ & 48.8 & 605.1508 & $299-461-503-543$ & $\mathrm{C}_{28} \mathrm{H}_{30} \mathrm{O}_{15}$ & 1.163 \\
\hline 34 & $\begin{array}{c}\text { Diosmetin-7-O- } \\
\text { neohesperidoside-O-HMG * }\end{array}$ & 49.1 & 751.2056 & $\begin{array}{l}299-461-607- \\
\quad 649-689\end{array}$ & $\mathrm{C}_{34} \mathrm{H}_{40} \mathrm{O}_{19}$ & -3.202 \\
\hline 36 & $\begin{array}{l}\text { Demethoxycentaureidin-7-O- } \\
\text { glucoside-O-HMG }{ }^{*}\end{array}$ & 50.9 & 635.1604 & $314-329-491-533$ & $\mathrm{C}_{29} \mathrm{H}_{32} \mathrm{O}_{16}$ & -0.261 \\
\hline \multirow[t]{3}{*}{49} & $\begin{array}{c}\text { Diosmetin-O-glucoside-O- } \\
\text { HMG }{ }^{* *}\end{array}$ & 50.5 & 605.1505 & 299-461-503-543 & $\mathrm{C}_{28} \mathrm{H}_{30} \mathrm{O}_{15}$ & 0.667 \\
\hline & Flavanones & & & & & \\
\hline & $\begin{array}{l}\text { Eriodictyol-di-O-glucoside-O- } \\
\text { HMG }{ }^{* *}\end{array}$ & 24.9 & 755.2003 & $\begin{array}{l}287-449-491- \\
531-593\end{array}$ & $\mathrm{C}_{33} \mathrm{H}_{40} \mathrm{O}_{20}$ & -3.469 \\
\hline 18 & $\begin{array}{l}\text { Neoeriocitrin-O-glucoside-O- } \\
\text { HMG }^{*}\end{array}$ & 28.0 & 901.2591 & $\begin{array}{c}\text { 287-595-637- } \\
\text { 377-739-757- } \\
799-839\end{array}$ & $\mathrm{C}_{39} \mathrm{H}_{50} \mathrm{O}_{24}$ & -1.918 \\
\hline \multirow[t]{2}{*}{20} & $\begin{array}{c}\text { Neohesperidin- } O \text {-glucoside- } O- \\
\text { HMG }\end{array}$ & 30.3 & 915.2731 & $\begin{array}{c}301-609-651- \\
691-771-813-853\end{array}$ & $\mathrm{C}_{40} \mathrm{H}_{52} \mathrm{O}_{24}$ & -3.724 \\
\hline & $\begin{array}{c}\text { Eriodictyol-O-glucoside-O- } \\
\mathrm{HMG}^{* *}\end{array}$ & 35.4 & 593.1495 & $287-449-491-531$ & $\mathrm{C}_{27} \mathrm{H}_{30} \mathrm{O}_{15}$ & -0.596 \\
\hline 24 & Eriocitrin-O-HMG ** & 35.8 & 739.2052 & $\begin{array}{l}287-433-595- \\
\quad 637-677\end{array}$ & $\mathrm{C}_{33} \mathrm{H}_{40} \mathrm{O}_{19}$ & -2.805 \\
\hline 27 & Neoeriocitrin-O-HMG * & 37.6 & 739.2067 & $\begin{array}{l}287-433-595- \\
\quad 637-677\end{array}$ & $\mathrm{C}_{33} \mathrm{H}_{40} \mathrm{O}_{19}$ & -1.766 \\
\hline 45 & Bergamjuicin glucoside & 40.6 & 1047.3167 & $\begin{array}{l}\text { 741-885-903- } \\
945-985\end{array}$ & $\mathrm{C}_{45} \mathrm{H}_{60} \mathrm{O}_{28}$ & -1.993 \\
\hline 46 & $\begin{array}{l}\text { Naringenin-O-rutinoside-O- } \\
\text { HMG ** }\end{array}$ & 43.9 & 723.2142 & $\begin{array}{l}271-417-579- \\
621-661\end{array}$ & $\mathrm{C}_{33} \mathrm{H}_{40} \mathrm{O}_{1}$ & 1.534 \\
\hline 30 & $\begin{array}{c}\text { Naringenin } \\
\text { 7-O-glucoside- } O-H M G *\end{array}$ & 44.8 & 577.1554 & $271-433-475-515$ & $\mathrm{C}_{27} \mathrm{H}_{30} \mathrm{O}_{14}$ & 0.378 \\
\hline 31 & Melitidin (Naringin-O-HMG) * & 45.7 & 723.2148 & $579-621-661$ & $\mathrm{C}_{33} \mathrm{H}_{40} \mathrm{O}_{1}$ & 2.364 \\
\hline 32 & $\begin{array}{c}\text { Hesperetin-O-glucoside- } O \text {-HMG } \\
\text { isomer } 1 \text { * }\end{array}$ & 47.1 & 607.1666 & $301-463-505-545$ & $\mathrm{C}_{28} \mathrm{H}_{31} \mathrm{O}_{15}$ & 1.406 \\
\hline 48 & $\begin{array}{l}\text { Hesperetin-O-rutinoside-O- } \\
\text { HMG }^{* *}\end{array}$ & 47.7 & 753.2221 & $301-609-651-691$ & $\mathrm{C}_{34} \mathrm{H}_{42} \mathrm{O}_{19}$ & -2.065 \\
\hline 33 & $\begin{array}{c}\text { Hesperetin-di- } O \text {-glucoside- } O \text { - } \\
\text { HMG ** }\end{array}$ & 48.9 & 769.2156 & $\begin{array}{l}301-463-625- \\
\quad 667-707\end{array}$ & $\mathrm{C}_{34} \mathrm{H}_{42} \mathrm{O}_{20}$ & -3.861 \\
\hline 34 & $\begin{array}{c}\text { Hesperetin-O-glucoside-O-HMG } \\
\text { isomer } 2 *\end{array}$ & 49.8 & 607.1647 & $301-463-505-545$ & $\mathrm{C}_{28} \mathrm{H}_{31} \mathrm{O}_{15}$ & -1.724 \\
\hline 35 & $\begin{array}{c}\text { Brutieridin } \\
(\text { Neohesperidin-O-HMG }) *\end{array}$ & 50.0 & 753.2223 & $609-651-691$ & $\mathrm{C}_{34} \mathrm{H}_{42} \mathrm{O}_{19}$ & -1.799 \\
\hline
\end{tabular}


Table 2. Cont.

\begin{tabular}{|c|c|c|c|c|c|c|}
\hline Peak & $\begin{array}{l}\text { Identification/Putative } \\
\text { Identification }\end{array}$ & RT (min) & $m / z$ & $\begin{array}{l}\text { MS/MS Ion } \\
\text { Fragments }\end{array}$ & $\begin{array}{l}\text { Molecular } \\
\text { Formula }\end{array}$ & $\Delta$ ppm \\
\hline & \multicolumn{6}{|c|}{ Non-phenolics } \\
\hline \multirow[t]{5}{*}{43} & $\begin{array}{l}\text { 6-(beta-D-glucopyranosyloxy)-4- } \\
\text { methoxy-5-benzofuranpropanoic } \\
\text { acid-O-HMG }\end{array}$ & 38.4 & 541.1556 & $\begin{array}{l}191-217-235- \\
397-439-479\end{array}$ & $\mathrm{C}_{24} \mathrm{H}_{30} \mathrm{O}_{14}$ & 0.773 \\
\hline & \multicolumn{6}{|c|}{ Unknown } \\
\hline & 315-glucoside-O-HMG (1) & 39.0 & 621.1450 & $\begin{array}{l}300-315-477- \\
519-559-579\end{array}$ & $\mathrm{C}_{28} \mathrm{H}_{30} \mathrm{O}_{16}$ & -0.018 \\
\hline & 315-glucoside-O-HMG (2) & 46.2 & 621.1451 & $\begin{array}{l}300-315-477- \\
519-559-579\end{array}$ & $\mathrm{C}_{28} \mathrm{H}_{30} \mathrm{O}_{16}$ & 0.143 \\
\hline & $\begin{array}{l}\text { 255-C-glucoside-O-rhamnoside- } \\
\text { O-HMG }\end{array}$ & 54.1 & 707.2159 & $\begin{array}{l}255-357-401- \\
563-605-645\end{array}$ & $\mathrm{C}_{33} \mathrm{H}_{40} \mathrm{O}_{17}$ & -3.218 \\
\hline 50 & 201-acetyl-glucosyl-O-HMG & 56.7 & 549.1600 & 201-243-405-447 & $\mathrm{C}_{26} \mathrm{H}_{30} \mathrm{O}_{13}$ & -0.487 \\
\hline
\end{tabular}

Table 3. Compounds putatively identified with the untargeted approach.

\begin{tabular}{|c|c|c|c|c|c|c|}
\hline Peak & Putative Identification & RT (min) & $m / z$ & $\begin{array}{l}\text { MS/MS Ion } \\
\text { Fragments }\end{array}$ & $\begin{array}{l}\text { Molecular } \\
\text { Formula }\end{array}$ & $\Delta \mathrm{ppm}$ \\
\hline \multicolumn{7}{|c|}{ Non-phenolics } \\
\hline 1 & Quinic acid & 2.2 & 191.0564 & $133-147$ & $\mathrm{C}_{7} \mathrm{H}_{12} \mathrm{O}_{6}$ & 1.385 \\
\hline 1 & HM̄G-glucoside & 2.5 & 323.0974 & $161-179$ & $\mathrm{C}_{12} \mathrm{H}_{20} \mathrm{O}_{10}$ & 0.393 \\
\hline 54 & $\begin{array}{c}\text { 6-(beta-D-glucopyranosyloxy)-5- } \\
\text { benzofuranpropanoic } \\
\text { acid }\end{array}$ & 19.1 & 367.1028 & $161-205$ & $\mathrm{C}_{17} \mathrm{H}_{20} \mathrm{O}_{9}$ & 0.481 \\
\hline 55 & $\begin{array}{l}\text { 6-(beta-D-glucopyranosyloxy)-4- } \\
\text { methoxy-5-benzofuranpropanoic } \\
\text { acid }\end{array}$ & 23.3 & 397.1135 & $176-191-217-235$ & $\mathrm{C}_{18} \mathrm{H}_{22} \mathrm{O}_{10}$ & 0.567 \\
\hline 58 & $\begin{array}{l}\text { 6-hydroxy-4-methoxy-5- } \\
\text { benzofuranpropanoic } \\
\text { acid }\end{array}$ & 41.5 & 235.0611 & $176-191$ & $\mathrm{C}_{12} \mathrm{H}_{12} \mathrm{O}_{5}$ & 1.010 \\
\hline 28 & Bergaptol & 42.1 & 201.0194 & 157 & $\mathrm{C}_{11} \mathrm{H}_{6} \mathrm{O}_{4}$ & 1.165 \\
\hline 62 & Deacetylnomilinic acid & 57.5 & 489.2125 & $325-333-411$ & $\mathrm{C}_{26} \mathrm{H}_{34} \mathrm{O}_{9}$ & 0.611 \\
\hline 63 & Limonoate A-ring lactone & 58.0 & 487.1953 & $383-427$ & $\mathrm{C}_{26} \mathrm{H}_{32} \mathrm{O}_{9}$ & -1.968 \\
\hline 42 & Deacetylnomilin & 60.5 & 471.2015 & $307-325-409$ & $\mathrm{C}_{26} \mathrm{H}_{32} \mathrm{O}_{8}$ & 0.330 \\
\hline \multicolumn{7}{|c|}{ Phenols } \\
\hline 10 & $\begin{array}{c}\text { p-Coumaric acid } \\
\text { 3-[2,4,5-trihydroxy-3-(3- }\end{array}$ & 13.8 & 163.0406 & 119 & $\mathrm{C}_{9} \mathrm{H}_{8} \mathrm{O}_{3}$ & 1.629 \\
\hline 59 & $\begin{array}{l}\text { methylbut-2-en-1- } \\
\text { yl)phenyl]propanoic } \\
\text { acid }\end{array}$ & 51.9 & 265.1072 & $\begin{array}{c}87-151-163-177- \\
185-203-221\end{array}$ & $\mathrm{C}_{14} \mathrm{H}_{18} \mathrm{O}_{5}$ & 0.100 \\
\hline \multicolumn{7}{|c|}{ Polyphenols } \\
\hline 8 & $\begin{array}{c}\text { Neoeriocitrin-O- } \\
\text { glucoside/eriocitrin-O-glucoside }\end{array}$ & 11.9 & 757.2160 & 287-449-595 & $\mathrm{C}_{33} \mathrm{H}_{42} \mathrm{O}_{20}$ & -3.394 \\
\hline 51 & $\begin{array}{l}\text { Luteolin-O-neohesperidoside- } O \text { - } \\
\text { glucoside }\end{array}$ & 15.5 & 755.2001 & $285-447-593$ & $\mathrm{C}_{33} \mathrm{H}_{40} \mathrm{O}_{20}$ & -3.694 \\
\hline 53 & $\begin{array}{l}\text { Luteolin-C-glucoside-O- } \\
\text { rhamnoside }\end{array}$ & 17.5 & 593.1502 & $285-447-473$ & $\mathrm{C}_{27} \mathrm{H}_{30} \mathrm{O}_{15}$ & 0.114 \\
\hline 55 & Luteolin-O-rutinoside & 23.5 & 593.1501 & 285 & $\mathrm{C}_{27} \mathrm{H}_{30} \mathrm{O}_{15}$ & -1.860 \\
\hline 56 & $\begin{array}{l}\text { Luteolin-O-neohesperidoside-O- } \\
\text { rhamnoside }\end{array}$ & 24.1 & 739.2050 & $285-593$ & $\mathrm{C}_{33} \mathrm{H}_{40} \mathrm{O}_{19}$ & -4.065 \\
\hline 18 & Hesperetin-di-C-glucoside & 27.4 & 625.1756 & $301-343-463-505$ & $\mathrm{C}_{28} \mathrm{H}_{34} \mathrm{O}_{16}$ & -2.892 \\
\hline 18 & $\begin{array}{l}\text { Naringenin-C-glucoside-di-O- } \\
\text { rhamnoside }\end{array}$ & 27.4 & 725.2299 & $271-459-605$ & $\mathrm{C}_{33} \mathrm{H}_{42} \mathrm{O}_{18}$ & 1.599 \\
\hline
\end{tabular}


Table 3. Cont

\begin{tabular}{|c|c|c|c|c|c|c|}
\hline Peak & Putative Identification & RT (min) & $m / z$ & $\begin{array}{l}\text { MS/MS Ion } \\
\text { Fragments }\end{array}$ & $\begin{array}{l}\text { Molecular } \\
\text { Formula }\end{array}$ & $\Delta \mathrm{ppm}$ \\
\hline 57 & Apigenin-O-glucoside & 31.9 & 431.0976 & 269 & $\mathrm{C}_{21} \mathrm{H}_{20} \mathrm{O}_{10}$ & 0.327 \\
\hline 43 & $\begin{array}{l}\text { Luteolin-O-acetyl-O- } \\
\text { neohesperidoside }\end{array}$ & 38.4 & 635.1610 & $285-327-489-593$ & $\mathrm{C}_{29} \mathrm{H}_{32} \mathrm{O}_{16}$ & 0.339 \\
\hline 46 & $\begin{array}{l}\text { Naringenin-C-glucoside-O- } \\
\text { acetyl-rhamnoside }\end{array}$ & 43.9 & 621.1812 & $\begin{array}{l}271-313-459- \\
501-579\end{array}$ & $\mathrm{C}_{29} \mathrm{H}_{34} \mathrm{O}_{15}$ & -0.316 \\
\hline \multirow[t]{2}{*}{33} & $\begin{array}{l}\text { Diosmetin-O-acetyl- } \\
\text { neohesperidoside }\end{array}$ & 48.8 & 649.1771 & 284-299-607 & $\mathrm{C}_{30} \mathrm{H}_{34} \mathrm{O}_{16}$ & 0.749 \\
\hline & Unknown & & & & & \\
\hline 52 & Unknown 1 & 15.8 & 611.1617 & $\begin{array}{l}287-329-373- \\
449-475-491\end{array}$ & $\mathrm{C}_{27} \mathrm{H}_{32} \mathrm{O}_{16}$ & 1.700 \\
\hline 36 & 255-C-glucoside-O-rhamnoside & 51.3 & 563.1769 & $\begin{array}{c}255-279-297- \\
401-443\end{array}$ & $\mathrm{C}_{27} \mathrm{H}_{32} \mathrm{O}_{13}$ & 1.745 \\
\hline 60 & Unknown 2 & 52.4 & 417.0819 & $\begin{array}{c}129-161-173- \\
189-251-277-295\end{array}$ & $\mathrm{C}_{20} \mathrm{H}_{18} \mathrm{O}_{10}$ & 0.227 \\
\hline 61 & Unknown 3 & 53.7 & 417.0816 & $\begin{array}{c}129-161-173- \\
189-251-277-295\end{array}$ & $\mathrm{C}_{20} \mathrm{H}_{18} \mathrm{O}_{10}$ & 0.017 \\
\hline
\end{tabular}

(a)

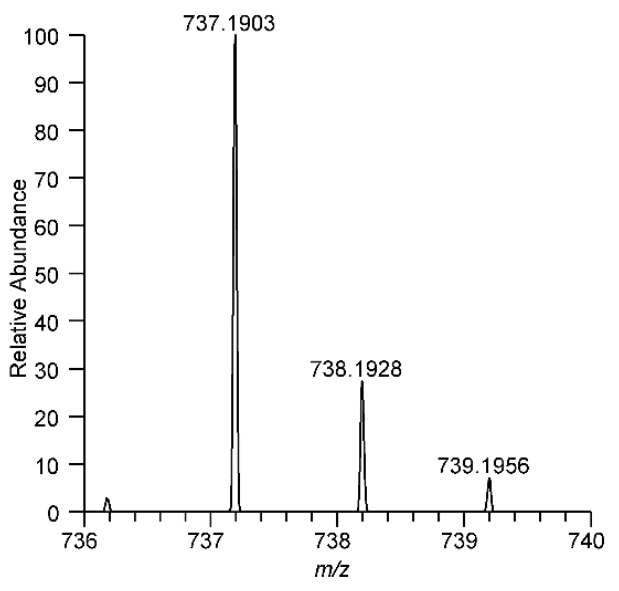

(b)

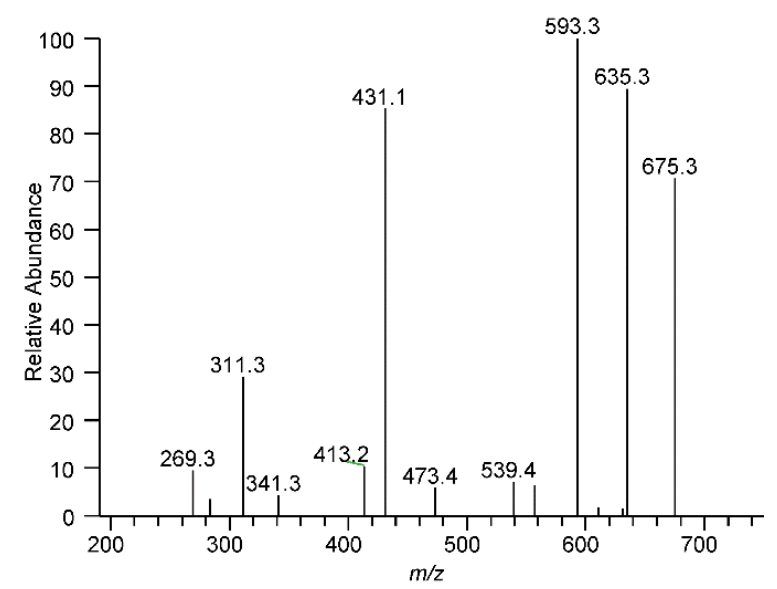

(c)

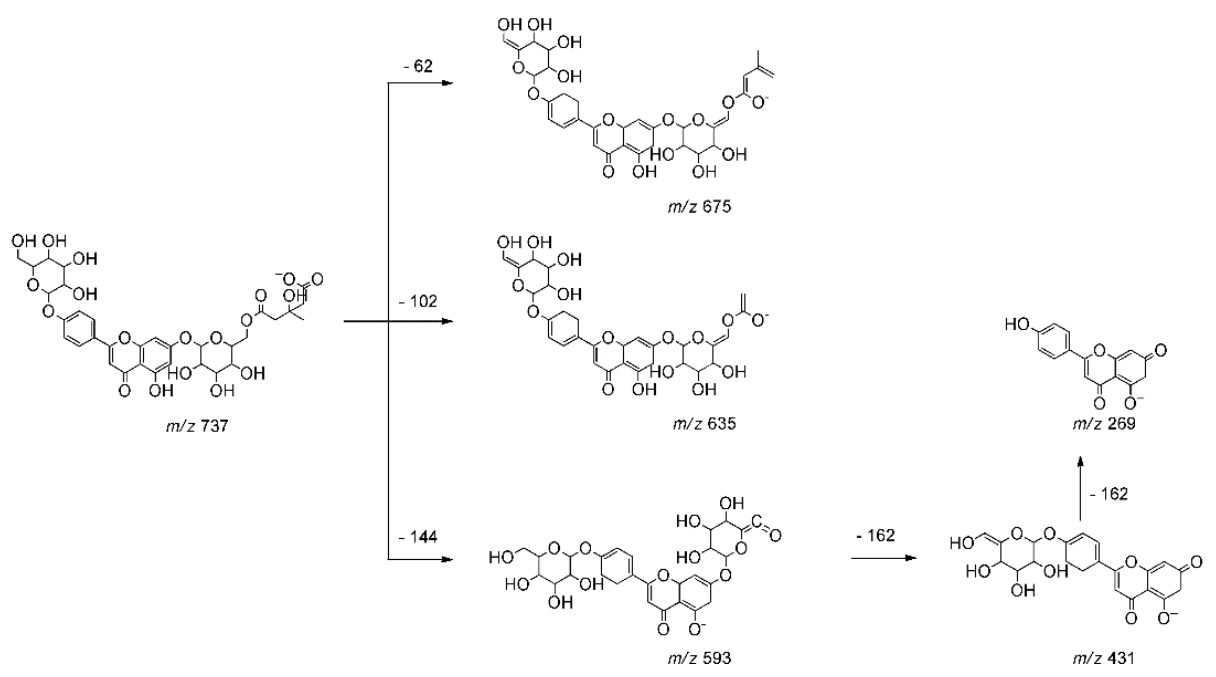

Figure 2. Identification of apigenin-di-O-glucoside-O-HMG by MS and MS/MS studies. Panel (a): full mass spectrum with accurate mass and isotopic pattern; panel (b): MS/MS spectrum; panel (c): proposed fragment tree MS/MS fragmentation. 


\subsection{Semi-Quantitative Analysis of the Identified Metabolites}

The identified metabolites are clustered into three main classes: phenols, polyphenols (flavones and flavanones) and "others", which include organic acids, limonoids, coumarins and unknowns. Figure 3 shows the relative content of each class calculated on the basis of the peak areas: BLPF comprises $1.8 \%$ phenols, $95.5 \%$ polyphenols ( $33.1 \%$ flavones and $64.2 \%$ flavanones) and $2.7 \%$ others; BFPF contains a higher percentage of phenols $(4.5 \%)$ and others $(15.7 \%)$, while polyphenols are $79.8 \%$ (21.6\% flavones and $58.2 \%$ flavanones). Table 4 reports the fold change ( $\log 2$ value) of the relative abundance of the 100 common compounds in BFPF versus BLPF and the statistical significance (- $\log p$ value). $41 \mathrm{com}-$ pounds were found to have a similar relative abundance (log2 fold change between -1 and 1), 31 with a relative abundance higher in BFPF (red region, log2 fold change $<-1$ ) and 28 lower (green region, $\log 2$ fold change $>1$ ) in respect to leaf extract. Figure 4 shows the data in a graphical form (Volcano plot).
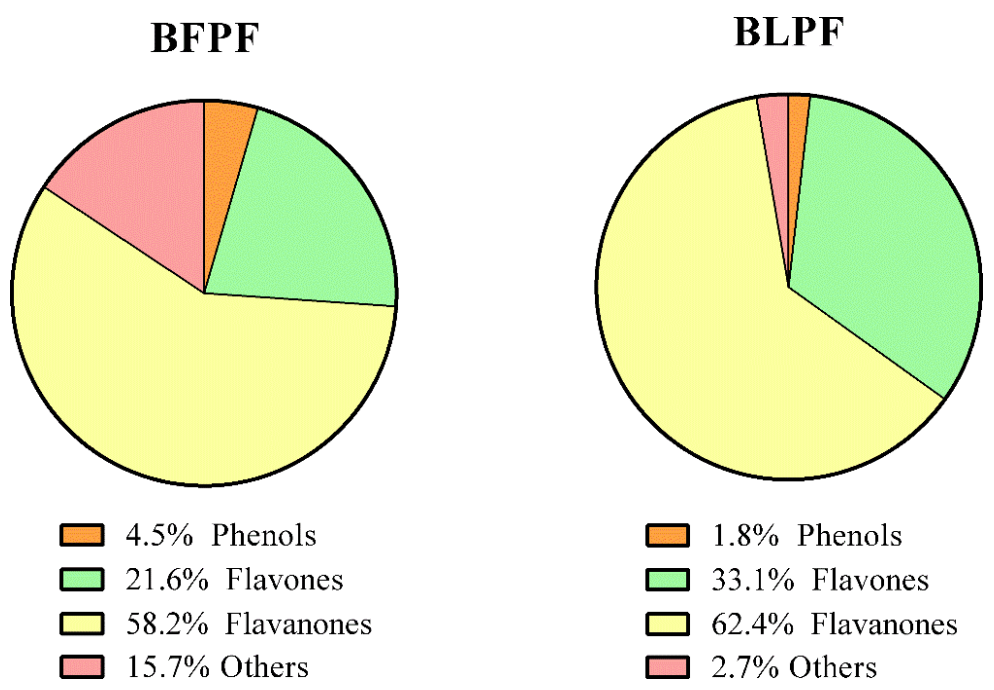

Figure 3. Percentage composition calculated on the base of peak areas of BLPF and BFPF. Flavones are in green, flavanones in yellow, phenols in orange and organic acids in red.

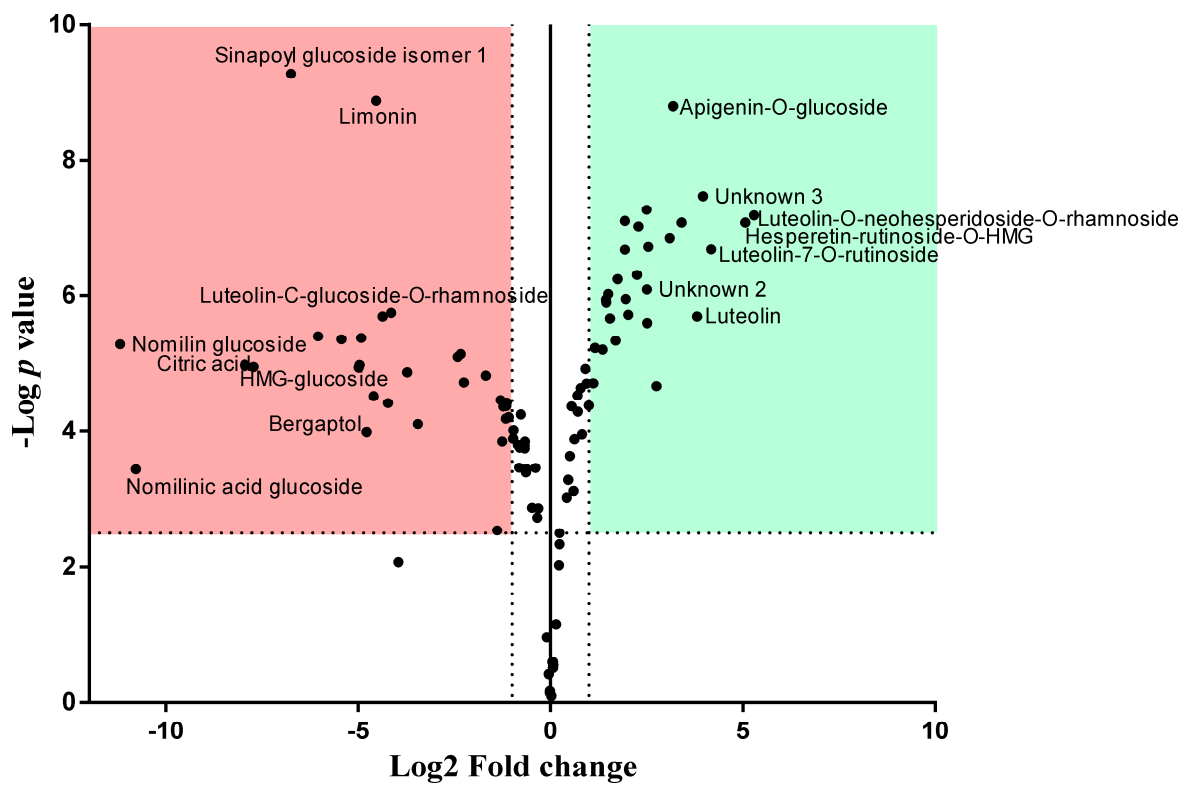

Figure 4. Volcano plot showing the semi-quantitative difference for each metabolite present in the two extracts. Those compounds significantly higher in BFPF are in red, while those significantly higher in BLPF are in green. The most statistically different compounds are named. 
Table 4. $\log 2$ Fold change and $-\log p$ values for the 100 common compounds of the two extracts.

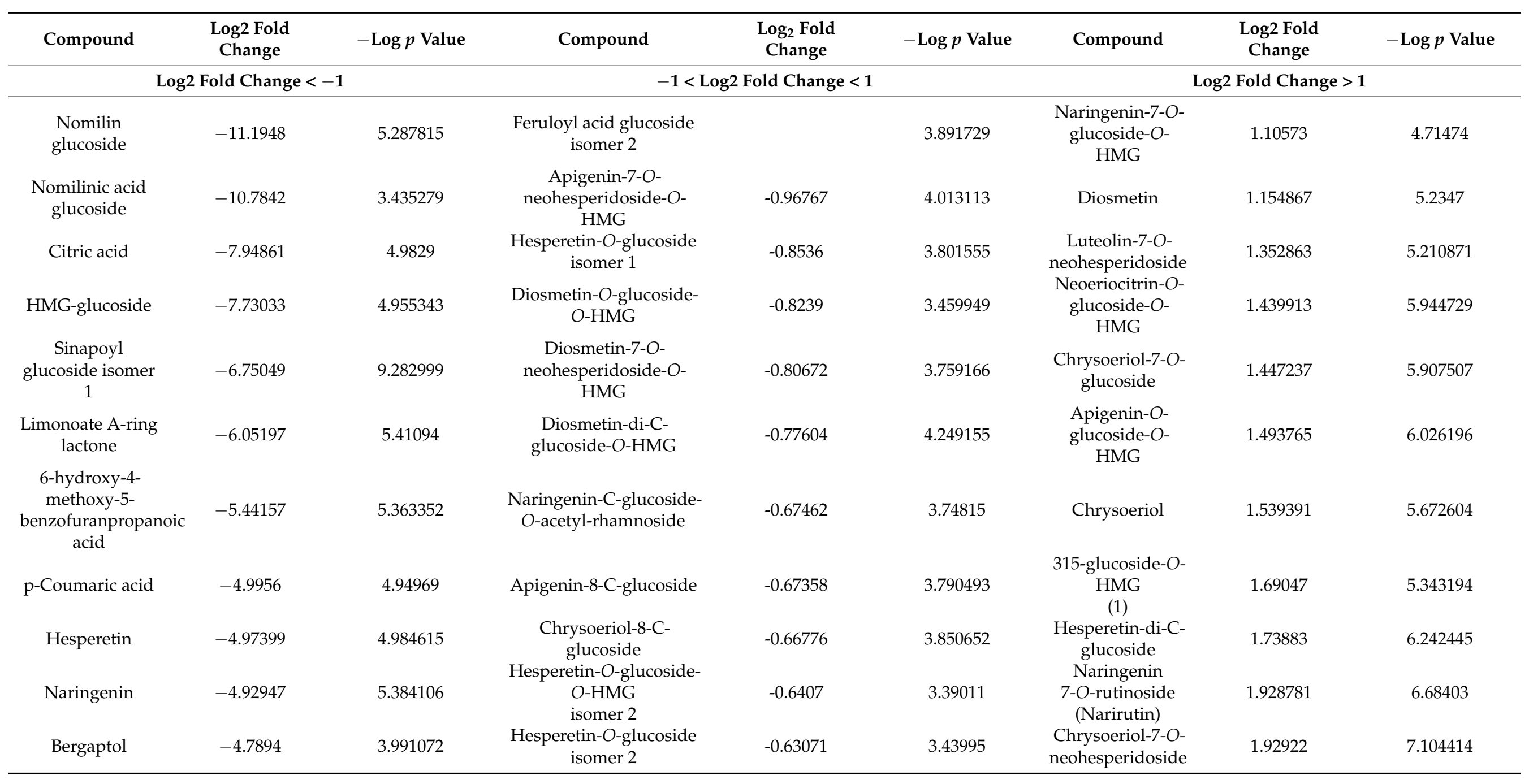


Table 4. Cont

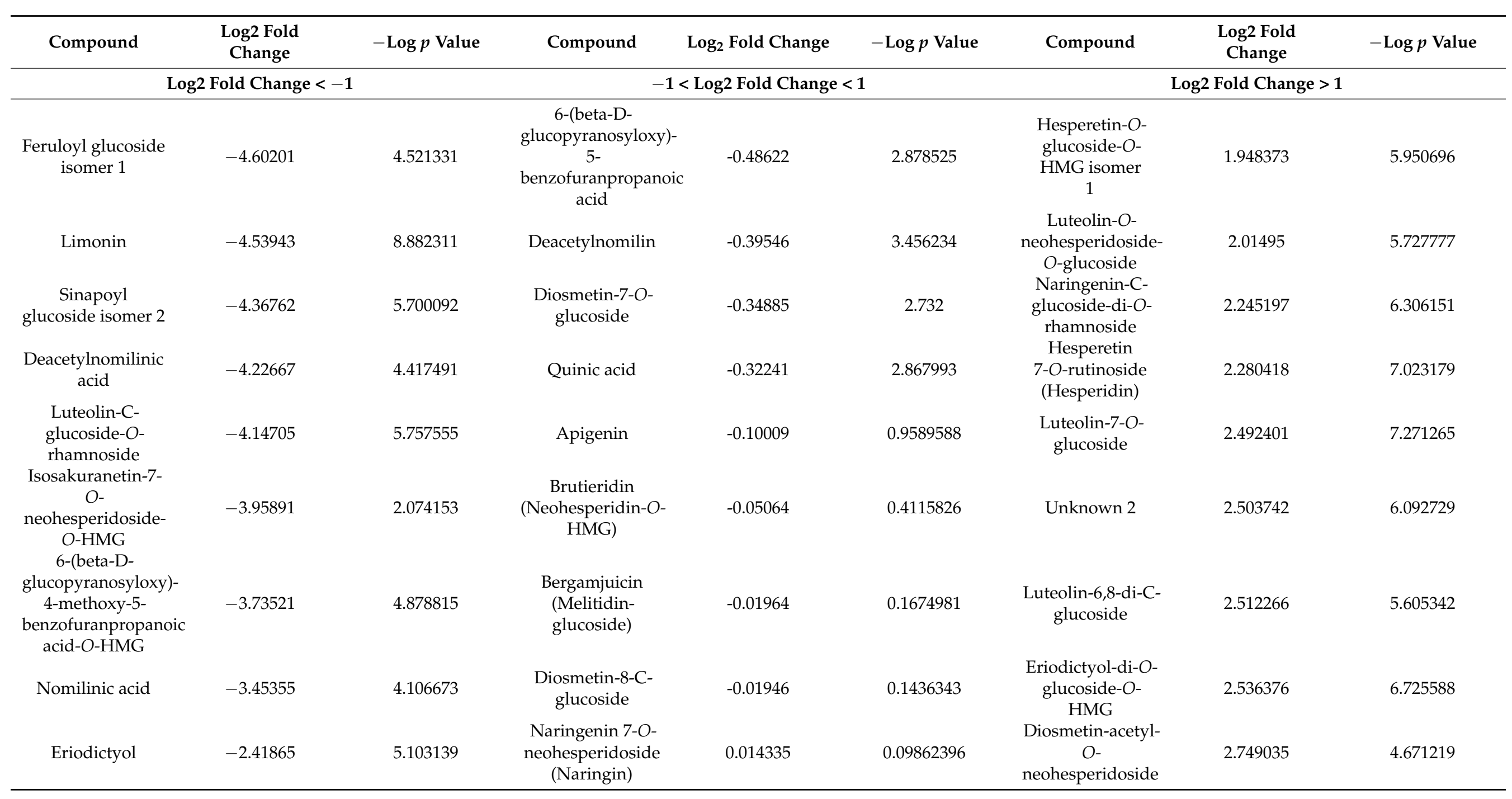


Table 4. Cont.

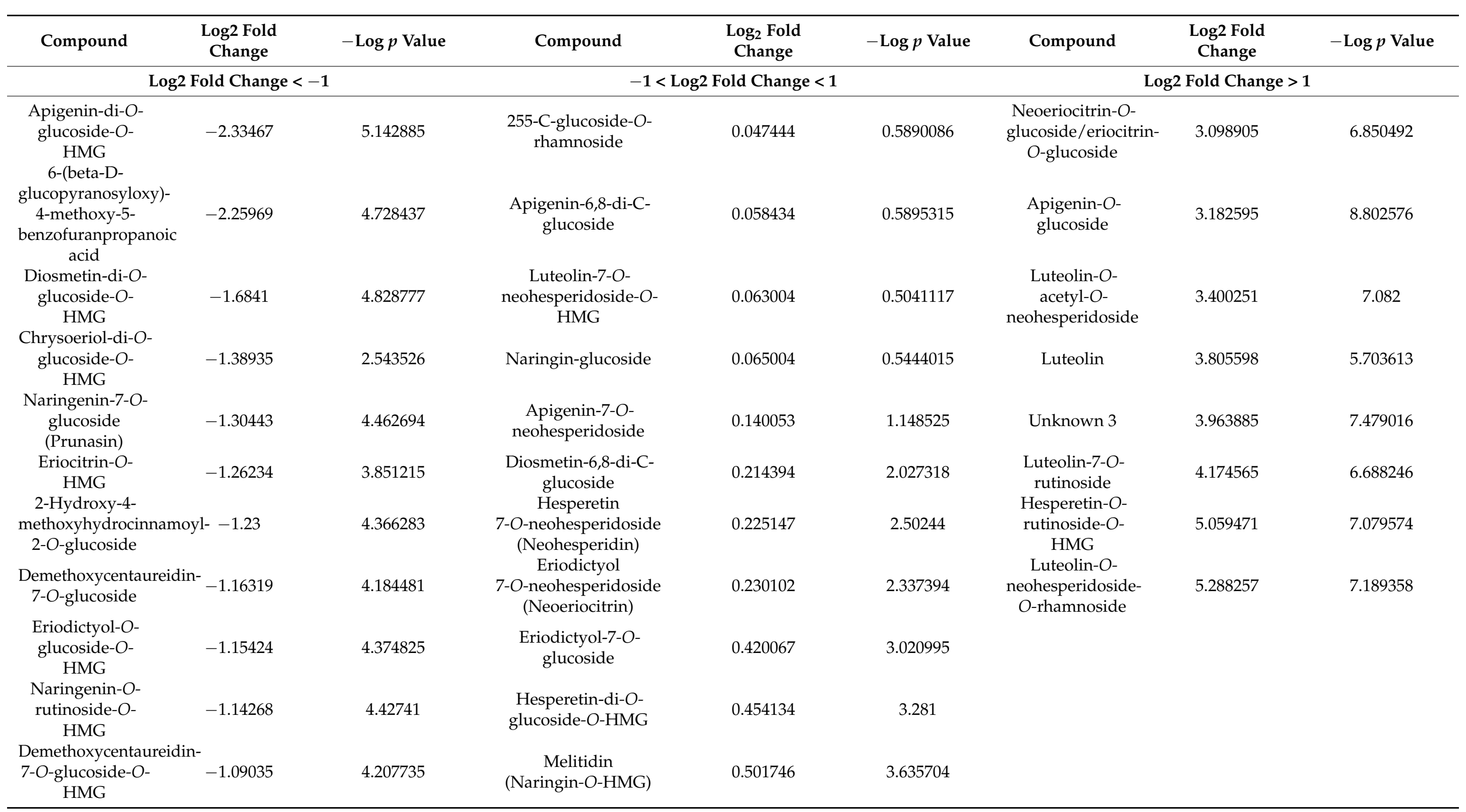


Table 4. Cont.

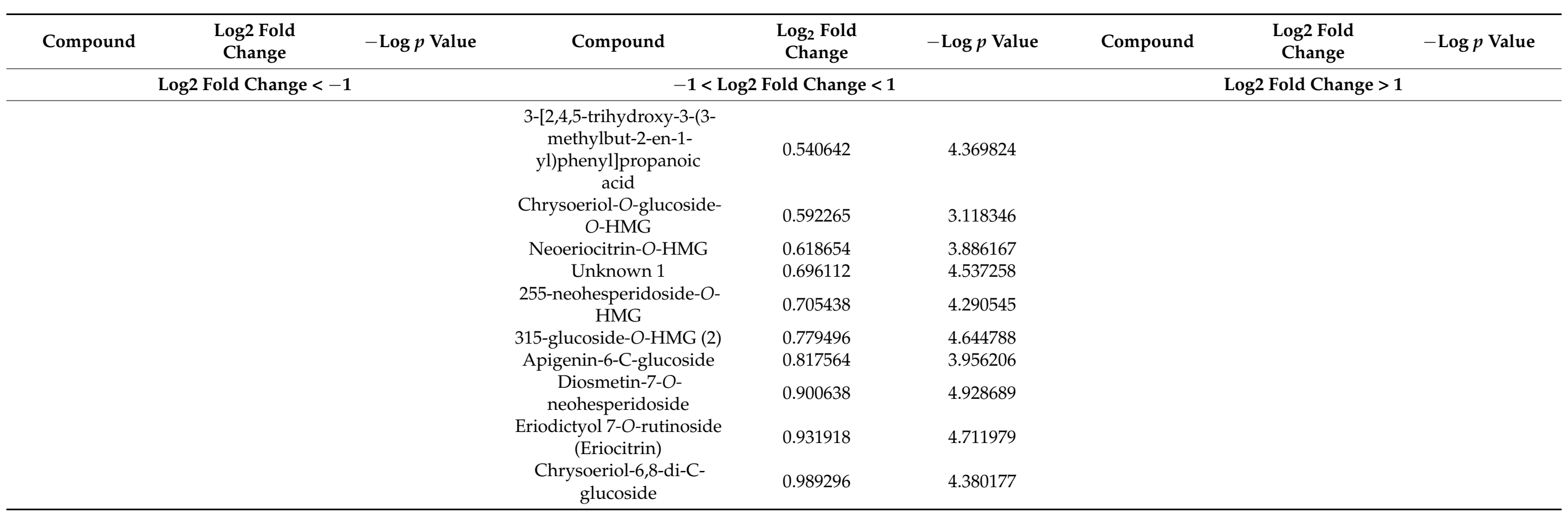


Figure 5 summarizes the qualitative differences between the two extracts as Venn diagrams: $92.6 \%$ of the compounds are in common (100), four compounds were found only in BFPF and 4 only in the leaf extract (Figure 5a). Regarding polyphenols, $71(94.7 \%)$ are present in both the extracts (Figure $5 b$ ) and, among these, all the 38 flavones identified in BFPF are also present in BLPF which contains two more (Figure $5 \mathrm{c}$ ), while $94.3 \%$ of the identified flavanones are in common (Figure 5d). Overall, we can say that the polyphenol pattern does not substantially differ.

(a)

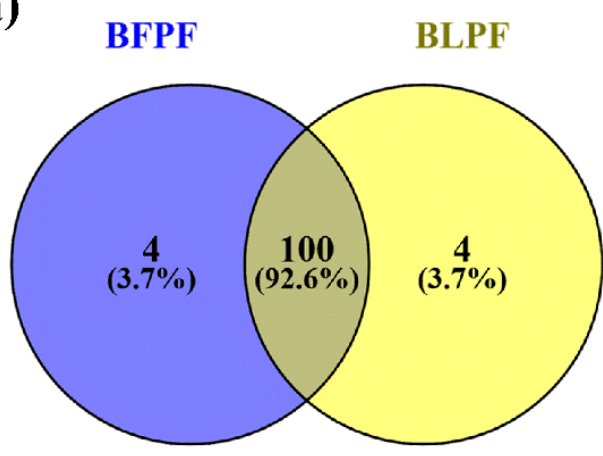

(c)

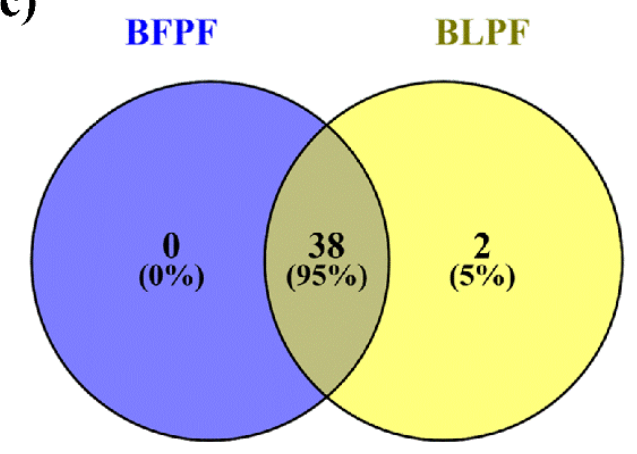

(b)

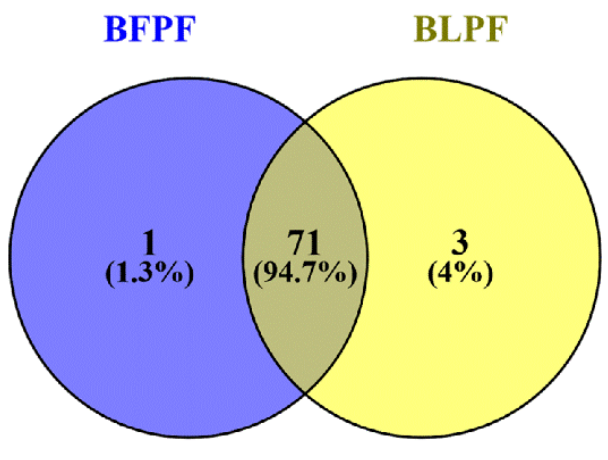

(d)

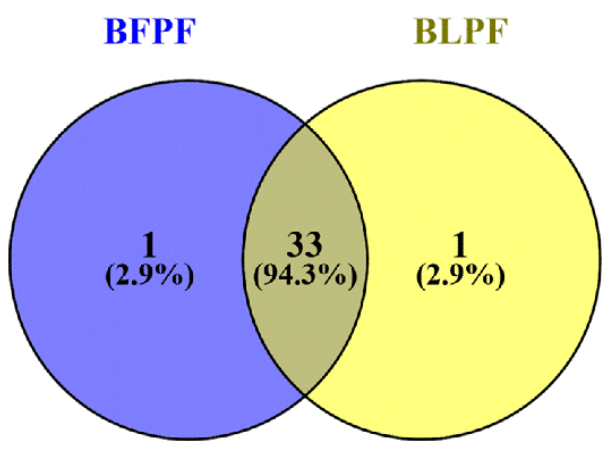

Figure 5. Venn diagrams showing the qualitative comparison between the two extracts: (a) of all the identified compounds, 100 (92.6\%) are in common between the two extracts; (b) $94.7 \%$ of the identified polyphenols are in common; (c) all the flavones identified in BFPF are also present in BLPF which contains 2 more flavones; (d) of the identified flavanones, 33 $(94.3 \%)$ are present in both the extract.

\subsection{Quantitative Analysis}

The quantitative analysis of the major flavonoids (neoeriocitrin, naringin, neohesperidin, melitidin, and brutieridin) present in bergamot extracts was performed by HPLCUV. Naringin was used as standard and the results are expressed as naringin equivalent (mg/g extract) (Table 5).

Table 5. Content (mg/g extract) of the major flavonoids present in the extracts expressed as mean (standard deviation).

\begin{tabular}{cccccc}
\hline Extract & Neoeriocitrin & Naringin & Neohesperidin & Melitidin & Brutieridin \\
\hline BFPF & $94.72(0.20)$ & $38.05(0.09)$ & $20.32(0.02)$ & $22.84(0.11)$ & $30.16(0.52)$ \\
BLPF & $133.42(3.22)$ & $164.31(1.48)$ & $144.95(1.36)$ & $31.2(2.19)$ & $59.77(2.04)$ \\
\hline
\end{tabular}

\subsection{Proanthocyanidin Analysis}

Proanthocyanidin were not detected in either the bergamot extracts by using both the methods reported in the method section. Results are reported in Figures S27-S30 of the Supplementary Materials. 


\subsection{Total Phenolic Content and Antioxidant Activity}

The mean values $( \pm \mathrm{SD})$ of the total phenolic content and the antioxidant activity measured with the ORAC, DPPH and ABTS assays are reported in Table 6, ascorbic acid values obtained from the literature are also reported as a reference [40-42]. The total phenolic content was higher in the leaf extract in respect to BFPF (by almost $38 \%$ ) and the difference was statistically different $(p=0.0328)$. Regarding the antioxidant activity, the ORAC value was higher in BLPF in respect to BFPF (a higher value indicates a higher antioxidant activity) but not statistically different $(p=0.331)$. When the ORAC values were normalized in respect to the polyphenol content, the values almost overlapped $(p=0.802)$. The antioxidant activity tested with DPPH was found to be higher for BLPF, showing a potency almost doubled in respect to BFPF and statistically different $(p<0.0001)$. The difference, although reduced, remained significant when the values were normalized in respect to the polyphenol content $(p=0.0232)$. The higher antioxidant activity of BLPF (almost double) was confirmed by the ABTS method ( $p=0.0012)$ but was not significantly different when normalized in respect to the polyphenol content $(p=0.0951)$.

Table 6. Total phenolic content and antioxidant activity mean values ( \pm SD) of the two tested extracts, absolute values and values normalized on total phenolic content.

\begin{tabular}{|c|c|c|c|c|}
\hline Extract & $\begin{array}{c}\text { Folin-Ciocalteu } \\
\text { mg Gallic } \\
\text { acid/g Extract }\end{array}$ & $\begin{array}{c}\text { ORAC } \\
\mu \mathrm{mol} \text { Trolox } / \mathrm{g} \\
\text { Extract }\end{array}$ & $\begin{array}{c}\text { DPPH } \\
\mathrm{IC}_{50}(\mu \mathrm{g} / \mathrm{mL})\end{array}$ & $\begin{array}{c}\text { ABTS } \\
\mathrm{IC}_{50}(\mu \mathrm{g} / \mathrm{mL})\end{array}$ \\
\hline BFPF & $167.3 \pm 12.5$ & $9834 \pm 542$ & $42.2 \pm 1.7$ & $96.3 \pm 7.3$ \\
\hline BLPF & $230.3 \pm 31.7$ & $13145 \pm 3487$ & $20.1 \pm 1.3$ & $49.5 \pm 5.6$ \\
\hline Ascorbic acid & & 4318 & 41.2 & 28.2 \\
\hline $\begin{array}{c}\text { Extract } \\
\text { Normalized } \\
\text { Values }\end{array}$ & & $\begin{array}{c}\text { Orac } \\
\mu \mathrm{mol} \text { Trolox/Mg } \\
\text { Gallic Acid }\end{array}$ & $\begin{array}{c}\text { DPPH } \\
\mathrm{IC}_{50}(\mu \mathrm{g} \text { Gallic } \\
\text { Acid } / \mathrm{mL})\end{array}$ & $\begin{array}{c}\text { ABTS } \\
\text { IC }_{50}(\mu g \text { Gallic } \\
\text { Acid } / \mathrm{mL})\end{array}$ \\
\hline $\begin{array}{l}\text { BFPF } \\
\text { BLPF }\end{array}$ & & $\begin{array}{l}63.6 \pm 11.2 \\
59.3 \pm 25.1\end{array}$ & $\begin{array}{c}7.07 \pm 0.74 \\
4.65 \pm 0.9\end{array}$ & $\begin{array}{l}16.2 \pm 2.4 \\
11.5 \pm 2.8\end{array}$ \\
\hline
\end{tabular}

\subsection{Anti-Inflammatory Activity}

To evaluate and compare the anti-inflammatory effect of the two extracts, R3/ 1 control cells were stably transduced with an NF-kB-driven reporter encoding for luciferase to generate R3/1-NF- $\mathrm{KB}$ cells. When stimulated with IL- $1 \alpha$, a potent inducer of the proinflammatory pathway through NF- $\kappa B$, transduced R3/1-NF- $\kappa B$ cells reported a luminescence signal increased more than 5 -fold in respect to control cells (Figure 6). The cell model was validated by using rosiglitazone which is a well-known anti-inflammatory agent via NF- $k$ B inhibition (Figure 6). The anti-inflammatory activity of the two extracts was tested by using two different protocols: in one case the pro-inflammatory agent IL-1 $\alpha$ was incubated in the presence of the extract while in the second case it was added after removing the extract. Both the extracts were found not to affect the cell viability in the concentration range $10-250 \mu \mathrm{g} / \mathrm{mL}$ as determined by the MTT assay (Figure 7). A dosedependent anti-inflammatory effect was observed for both the extracts. As expected, the anti-inflammatory activity was higher when IL- $1 \alpha$ was incubated in the presence of the extract: in such a condition both the extracts were found to be already active at $10 \mu \mathrm{g} / \mathrm{mL}$ while when the extracts were removed, the activity was significant in a concentration range between 100 and $250 \mu \mathrm{g} / \mathrm{mL}$ for BLPF and at $250 \mu \mathrm{g} / \mathrm{mL}$ for BFPF (Figure 8). BLPF was found to be significantly more effective in respect to BFPF at $250 \mu \mathrm{g} / \mathrm{mL}$ when the extract was removed and at all the concentrations tested in the second cell experiment. 
(a)

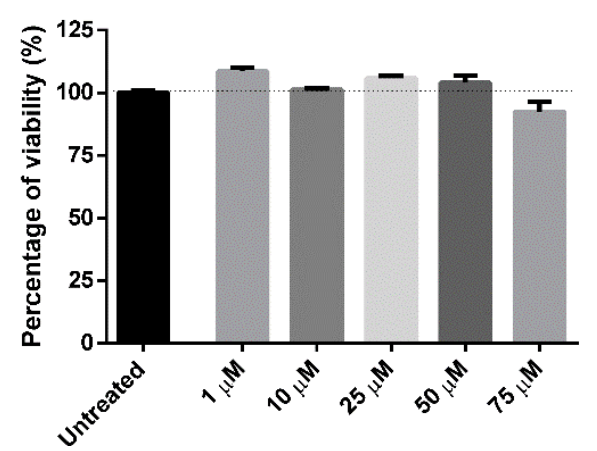

(b)

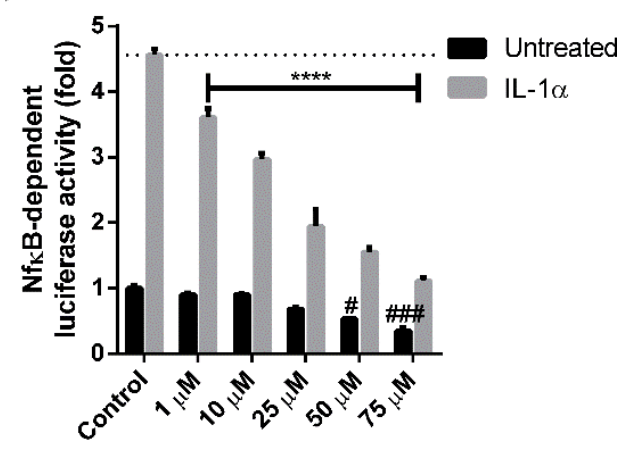

Figure 6. Dose-dependent effect of rosiglitazone used as reference active compound on R3/1-NF-kB cells (a) viability (MTT assay) and (b) IL-1 $\alpha$ induced NF- $\mathrm{kB}$ activation. Data are reported as mean \pm SEM. The statistical significance difference of each concentration in respect to the control was analyzed by ONE-WAY analysis followed by the Bonferroni post-test. For the untreated series: ${ }^{\#} p<0.05,{ }^{\# \#} p<0.001$. For IL-1 $\alpha$ treated samples: ${ }^{* * * *} p<0.0001$.

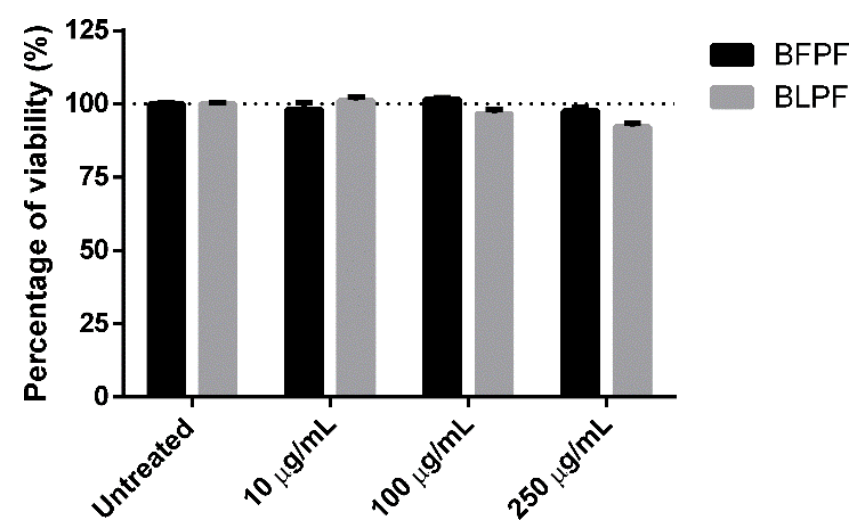

Figure 7. Dose-dependent effect of BFPF and BLPF on cell viability as measured by MTT on R3/1NF- $\mathrm{KB}$ cells. Data are shown as mean \pm SEM.

(a)

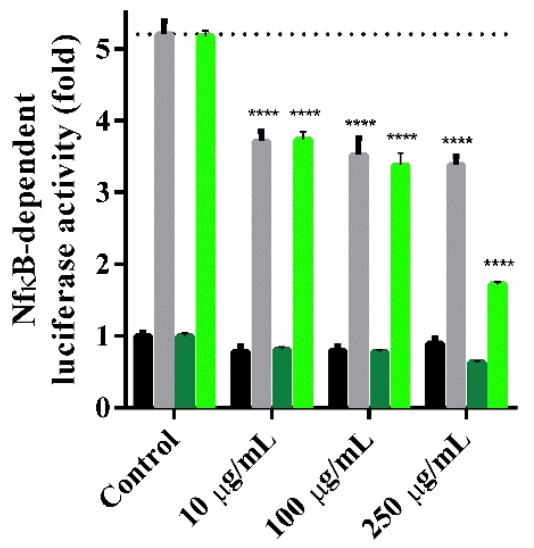

(b)

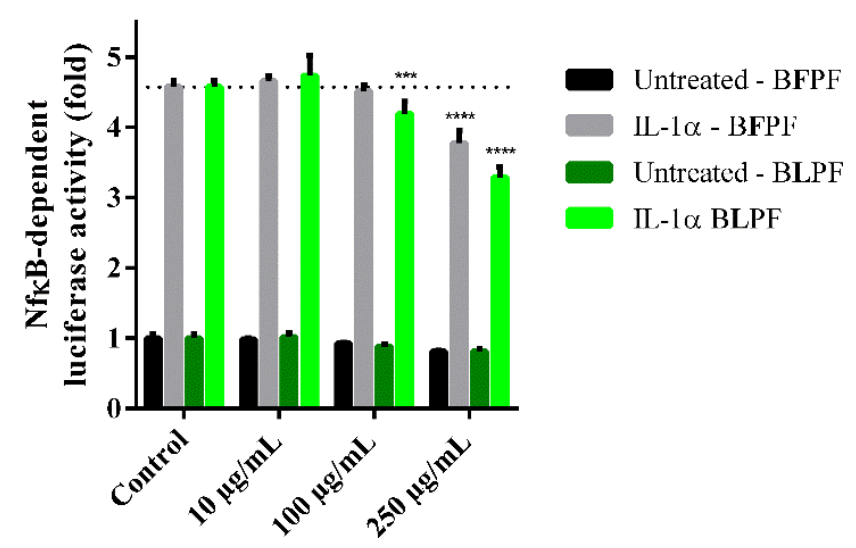

Figure 8. Dose-dependent effect of BFPF and BLPF on IL-1 $\alpha$ induced NF- $\mathrm{kB}$ activation. R3/1-NF-kB cells were incubated for $18 \mathrm{~h}$ with different concentrations of BLPF (green) or BFPF (grey), followed by a $6 \mathrm{~h}$ stimulation with IL- $1 \alpha$, without the removal of medium containing the extract (a) or with the removal (b). Then NF- $\mathrm{kB}$ dependent luciferase activity was measured. Data are reported as mean \pm SEM. The statistical significance difference of each concentration of BFPF and BLPF in respect to the control was analyzed by ONE-WAY analysis followed by the Bonferroni post-test. ${ }^{* * *} p<0.001$ and ${ }^{* * * *} p<$ 0.0001 . 


\section{Discussion}

By using targeted, semi-targeted and untargeted approaches, the qualitative and semi-quantitative profiling of the enriched polyphenol fraction obtained from bergamot leaves and fruit was compared. Overall, the qualitative composition is quite overlapping since 71 polyphenolic components were found in both the extracts, accounting for $98.7 \%$ and $96 \%$ of the total polyphenols present in leaf and fruit, respectively. Some polyphenols were only found in fruit extract and others only in leaf extract, accounting for 1.3 and $4 \%$, respectively. Regarding the quantitative aspect, it was possible to compare by a semi-quantitative approach the polyphenol content of the two extracts due to the fact that the same method for polyphenol enrichment was used. The total polyphenol content was 38\% higher in leaf in respect to fruit, suggesting a higher content of polyphenols in the starting raw material. The non-polyphenolic constituents were also addressed as summarized in Table 1 (analytes are classified as polyphenols, phenols and non-phenolics). The higher polyphenolic content in the leaf correlates to the higher relative content of the non-polyphenolic constituents in BFPF in respect to BLPF. Organic acids and non-phenolic constituents (others) account in BFPF for $4.5 \%$ and $15.7 \%$, respectively, (sum of $20.2 \%$ ) as opposed to $1.8 \%$ and $2.7 \%$ in leaf (sum of $4.5 \%$ ). By contrast, polyphenols, and in particular flavanones and flavones, are lower in BFPF in respect to BLPF, being $21.6 \%$ and $58.2 \%$ $(79,8 \%)$ as opposed to $33.1 \%$ and $62.4 \%$ (sum of $95.5 \%$ ), respectively. Hence the polyphenolic qualitative composition is quite overlapping in the two extracts while the relative content is higher in leaf and the non-polyphenolic amount is higher in the fruit. Besides comparing the extracts, and profiling for the first time the bergamot leaf composition, in the present study compounds not previously reported in bergamot were identified in BFPF, whose biological and pharmacological activity is emerging. In particular, by using a semi-targeted approach aimed at identifying the HMG-derivatives, 21 more components carrying the HMG moiety were identified. These compounds deserve further investigation since the cholesterol lowering effect of bergamot polyphenol extract has been attributed to the modulation of the key enzyme 3-hydroxy-3-methylglutaryl-CoA reductase (HMGR). In particular, computational studies have suggested that HMG derivatives have the ability to replace HMG-CoA, the endogenous substrate of HMG-CoA reductase [11]. Such a mechanism has been proposed to explain the ability of BPF to inhibit cholesterol synthesis and in particular to act as a cholesterol-lowering food supplement as demonstrated in intervention studies performed on metabolic syndrome patients. The identification of other HMG derivatives further improves our understanding of bergamot's cholesterol lipid lowering effect and would permit the investigation of the effect of these identified molecules on HMGR by using different approaches such as molecular docking which is currently applied in our lab. HMG derivatives were also identified in leaves and the profile is quite overlapping, but two more HMG components were detected, for which an annotation was possible only for luteolin-O-glucoside-O-HMG, while for the compounds at $\mathrm{m} / \mathrm{z} 549.1600$ we were not able to give a hypothesis of characterization. Bergamjuicin glucoside was instead putatively identified only in BFPF. By using an untargeted approach, we then searched for other compounds in BPF in respect to those reported in the literature. We found 26 more compounds as shown in Table 3. The higher polyphenol content in leaves in respect to the fruit partially explains the higher antioxidant activity as determined by three different methods, ORAC, DPPH and ABTS. In particular, the antioxidant activity of BLPF was found to be higher than that of fruit in all the three methods and accounts for $34 \%, 52 \%$ and $48 \%$, respectively. The difference was found to be significant for DPPH and ABTS but not for ORAC, probably due to the limited precision of this method $(\mathrm{CV} \%, 26 \%)$ in respect to the two others. By normalizing the antioxidant activity in respect to the polyphenol content, the differences in antioxidant activities between the two extracts greatly reduced $(7 \%, 34 \%, 29 \%$ for the ORAC, DPPH and ABTS) and were found significantly different only for the ABTS method. The data suggest that the higher antioxidant activity found in leaves in respect to the BPF is due to their different polyphenol content and not to a different composition and this is confirmed because they become similar when normalized 
on the basis of the polyphenol content. Compared to known antioxidants the two extracts showed a good antioxidant activity: ascorbic acid is reported to have $4318 \mathrm{TE} / \mathrm{g}$ as ORAC value [40], a $\mathrm{IC}_{50}$ mean value of $41.2 \mu \mathrm{g} / \mathrm{mL}$ for the DPPH assay [41], and a $\mathrm{IC}_{50}$ value of $28.2 \mu \mathrm{g} / \mathrm{mL}$ for the ABTS assay [42]. Finally, we evaluated the effects of leaf and fruit on cell inflammation. BFPF has been reported to possess a significant anti-inflammatory activity in both in vitro and in vivo models through both an antioxidant effect and by a mechanism involving the inhibition of NF- $\kappa$ B activation. Such anti-inflammatory activity has been addressed to flavanone and flavone glycosides, as well as to their aglycones, including naringin and hesperidin, diosmetin, apigenin, and luteolin glycosides, which are contained in both leaf and fruit extracts. The two extracts were both found effective when incubated and pre-incubated with the inflammatory stimulus although they are more effective in the former condition. In both the experiments leaf extract was significantly more effective than the fruit extract in accordance with its higher antioxidant activity and higher polyphenol content. Hence the data confirm that the anti-inflammatory activity is due to the polyphenol fraction which is higher in leaves in respect to BFPF.

In conclusion, the comparison of the qualitative and quantitative profile of polyphenols as well as of the antioxidant and anti-inflammatory activity of bergamot leaf and fruit well indicates that leaf is a valid source of bergamot polyphenol extraction and even a richer source of polyphenol in respect to the fruit. The similar qualitative pattern of polyphenol components and of HMG derivatives suggest that leaf extract should possess a similar pharmacological activity to that of BFPF which needs to be confirmed in animal studies.

Supplementary Materials: The following are available online at https://www.mdpi.com/2076-3 921/10/2/141/s1, Method S1: Method validation, Table S1: Database for the targeted analysis, Figure S1: Quinic acid, Figure S2: HMG-O-glucoside, Figure S3: 6-(beta-D-glucopyranosyloxy)-5benzofuranpropanoic acid, Figure S4: 6-(beta-D-glucopyranosyloxy)-4-methoxy-5-benzofuranpropanoic acid, Figure S5: 6-hydroxy-4-methoxy-5-benzofuranpropanoic acid, Figure S6: Bergaptol, Figure S7: Deacetylnomilinic acid, Figure S8: Limonoate A-ring lactone, Figure S9: Deacetylnomilin, Figure S10: p-coumaric acid, Figure S11: 3-[2,4,5-trihydroxy-3-(3-methylbut-2-en-1-yl)phenyl]propanoic acid, Figure S12: Neoeriocitrin-O-glucoside/eriocitrin-O-glucoside, Figure S13: Luteolin-O-neohesperidosideO-glucoside, Figure S14: Luteolin-C-glucoside-O-rhamnoside, Figure S15: Luteolin-O-rutinoside, Figure S16: Luteolin-O-neohesperidoside-O-rhamnoside, Figure S17: Hesperetin-di-C-glucoside, Figure S18: Naringenin-C-glucoside-di-O-rhamnoside, Figure S19: Apigenin-O-glucoside, Figure S20: Luteolin-O-acetyl-O-neohesperidoside, Figure S21: Naringenin-C-glucoside-di-O-rhamnoside, Figure S22: Diosmetin-O-acetyl-neohesperidoside, Figure S23: Unknown 1, Figure S24: 255-Cglucoside-O-rhamnoside, Figure S25: Unknown 2, Figure S26: Unknown 3, Figure S27: UV trace of the LeucoselectTM GPC, Figure S28: UV trace of the BFPF GPC, Figure S29. UV trace of the BLPF GPC, Figure S30: Evaluation of tannin effect.

Author Contributions: Conceptualization, E.B. and G.A.; methodology, G.B., A.A., M.M., S.M., L.F., G.A., A.R., L.M., G.T., L.T., E.L., D.M., E.J.; formal analysis, G.B., A.A., M.M., J.L.G., S.M., L.F., G.T.; investigation, G.B., A.A., G.A., E.B., A.R., L.M.; resources, A.R., L.M., G.T., L.T., E.L., D.M., E.B.; writing一original draft preparation, G.B., G.A.; writing—review and editing, J.L.G., C.C., A.R., L.M., V.M., M.C., E.B., G.A.; supervision, V.M., E.B., G.A.; project administration, G.A. All authors have read and agreed to the published version of the manuscript.

Funding: This work has been partially supported by PON-MIUR 03PE00_78.

Institutional Review Board Statement: Not applicable.

Informed Consent Statement: Not applicable.

Data Availability Statement: The data presented in this study are available on request from the corresponding author. The data are not publicly available.

Acknowledgments: We acknowledge support by the Open APC European Project fund of the Università degli Studi di Milano. 
Conflicts of Interest: The funders had no role in the design of the study; in the collection, analyses, or interpretation of data; in the writing of the manuscript, or in the decision to publish the results. G.T., L.T., E.L., D.M. are employees of H\&AD Srl., a company which produces and commercializes $\mathrm{BPF}^{\circledR}$, a Bergamot Polyphenolic Fraction.

\section{References}

1. Cautela, D.; Vella, F.M.; Laratta, B. The Effect of Processing Methods on Phytochemical Composition in Bergamot Juice. Foods 2019, 8, 474. [CrossRef]

2. Mannucci, C.; Navarra, M.; Calapai, F.; Squeri, R.; Gangemi, S.; Calapai, G. Clinical Pharmacology of Citrus bergamia: A Systematic Review. Phytother. Res. 2017, 31, 27-39. [CrossRef] [PubMed]

3. Giuffrè, A.M. Bergamot (Citrus bergamia, Risso): The Effects of Cultivar and Harvest Date on Functional Properties of Juice and Cloudy Juice. Antioxidants 2019, 8, 221. [CrossRef] [PubMed]

4. Carresi, C.; Gliozzi, M.; Musolino, V.; Scicchitano, M.; Scarano, F.; Bosco, F.; Nucera, S.; Maiuolo, J.; Macrì, R.; Ruga, S.; et al. The Effect of Natural Antioxidants in the Development of Metabolic Syndrome: Focus on Bergamot Polyphenolic Fraction. Nutrients 2020, 12, 1504. [CrossRef] [PubMed]

5. Lamiquiz-Moneo, I.; Giné-González, J.; Alisente, S.; Bea, A.M.; Pérez-Calahorra, S.; Marco-Benedí, V.; Baila-Rueda, L.; Jarauta, E.; Cenarro, A.; Civeira, F.; et al. Effect of bergamot on lipid profile in humans: A systematic review. Crit. Rev. Food Sci. Nutr. 2020, 60, 3133-3143. [CrossRef]

6. Nauman, M.C.; Johnson, J.J. Clinical application of bergamot (Citrus bergamia) for reducing high cholesterol and cardiovascular disease markers. Integr. Food Nutr. Metab. 2019, 6, 249. [CrossRef]

7. Mollace, V.; Sacco, I.; Janda, E.; Malara, C.; Ventrice, D.; Colica, C.; Visalli, V.; Muscoli, S.; Ragusa, S.; Muscoli, C.; et al. Hypolipemic and hypoglycaemic activity of bergamot polyphenols: From animal models to human studies. Fitoterapia 2011, 82, 309-316. [CrossRef]

8. Musolino, V.; Gliozzi, M.; Scarano, F.; Bosco, F.; Scicchitano, M.; Nucera, S.; Carresi, C.; Ruga, S.; Zito, M.C.; Maiuolo, J.; et al. Bergamot Polyphenols Improve Dyslipidemia and Pathophysiological Features in a Mouse Model of Non-Alcoholic Fatty Liver Disease. Sci. Rep. 2020, 10, 2565. [CrossRef]

9. De Leo, M.; Piragine, E.; Pirone, A.; Braca, A.; Pistelli, L.; Calderone, V.; Miragliotta, V.; Testai, L. Protective Effects of Bergamot (Citrus bergamia Risso \& Poiteau) Juice in Rats Fed with High-Fat Diet. Planta Med. 2020, 86, 180-189. [CrossRef]

10. Mollace, V.; Scicchitano, M.; Paone, S.; Casale, F.; Calandruccio, C.; Gliozzi, M.; Musolino, V.; Carresi, C.; Maiuolo, J.; Nucera, S.; et al. Hypoglycemic and Hypolipemic Effects of a New Lecithin Formulation of Bergamot Polyphenolic Fraction: A Double Blind, Randomized, Placebo Controlled Study. Endocr. Metab. Immune Disord. Drug Targets 2019, 19, 136-143. [CrossRef]

11. Leopoldini, M.; Malaj, N.; Toscano, M.; Sindona, G.; Russo, N. On the inhibitor effects of bergamot juice flavonoids binding to the 3-hydroxy-3-methylglutaryl-CoA reductase (HMGR) enzyme. J. Agric. Food Chem. 2010, 58, 10768-10773. [CrossRef] [PubMed]

12. Ballistreri, G.; Amenta, M.; Fabroni, S.; Consoli, V.; Grosso, S.; Vanella, L.; Sorrenti, V.; Rapisarda, P. Evaluation of lipid and cholesterol-lowering effect of bioflavonoids from bergamot extract. Nat. Prod. Res. 2020, 22, 1-6. [CrossRef] [PubMed]

13. Ferlemi, A.V.; Lamari, F.N. Berry Leaves: An Alternative Source of Bioactive Natural Products of Nutritional and Medicinal Value. Antioxidants 2016, 5, 17. [CrossRef] [PubMed]

14. Baron, G.; Altomare, A.; Regazzoni, L.; Fumagalli, L.; Artasensi, A.; Borghi, E.; Ottaviano, E.; Del Bo, C.; Riso, P.; Allegrini, P.; et al. Profiling Vaccinium macrocarpon components and metabolites in human urine and the urine ex-vivo effect on Candida albicans adhesion and biofilm-formation. Biochem. Pharmacol. 2020, 173, 113726. [CrossRef]

15. Risitano, R.; Currò, M.; Cirmi, S.; Ferlazzo, N.; Campiglia, P.; Caccamo, D.; Ientile, R.; Navarra, M. Flavonoid fraction of Bergamot juice reduces LPS-induced inflammatory response through SIRT1-mediated NF-kB inhibition in THP-1 monocytes. PLoS ONE 2014, 9, e107431. [CrossRef]

16. Janda, E.; Salerno, R.; Martino, C.; Lascala, A.; La Russa, D.; Oliverio, M. Qualitative and quantitative analysis of the proautophagic activity of Citrus flavonoids from Bergamot Polyphenol Fraction. Data Brief 2018, 19, 1327-1334. [CrossRef]

17. Mandalari, G.; Bennett, R.N.; Bisignano, G.; Saija, A.; Dugo, G.; Lo Curto, R.B.; Faulds, C.B.; Waldron, K.W. Characterization of flavonoids and pectins from bergamot (Citrus bergamia Risso) peel, a major byproduct of essential oil extraction. J. Agric. Food Chem. 2006, 54, 197-203. [CrossRef]

18. Gattuso, G.; Barreca, D.; Caristi, C.; Gargiulli, C.; Leuzzi, U. Distribution of flavonoids and furocoumarins in juices from cultivars of Citrus bergamia Risso. J. Agric. Food Chem. 2007, 55, 9921-9927. [CrossRef]

19. Gattuso, G.; Caristi, C.; Gargiulli, C.; Bellocco, E.; Toscano, G.; Leuzzi, U. Flavonoid glycosides in bergamot juice (Citrus bergamia Risso). J. Agric. Food Chem. 2006, 54, 3929-3935. [CrossRef]

20. Da Pozzo, E.; De Leo, M.; Faraone, I.; Milella, L.; Cavallini, C.; Piragine, E.; Testai, L.; Calderone, V.; Pistelli, L.; Braca, A.; et al. Antioxidant and Antisenescence Effects of Bergamot Juice. Oxidative Med. Cell. Longev. 2018, 2018, 9395804. [CrossRef]

21. Gardana, C.; Nalin, F.; Simonetti, P. Evaluation of flavonoids and furanocoumarins from Citrus bergamia (Bergamot) juice and identification of new compounds. Molecules 2008, 13, 2220-2228. [CrossRef] [PubMed]

22. Spigoni, V.; Mena, P.; Fantuzzi, F.; Tassotti, M.; Brighenti, F.; Bonadonna, R.C.; Del Rio, D.; Dei Cas, A. Bioavailability of Bergamot (Citrus bergamia) Flavanones and Biological Activity of Their Circulating Metabolites in Human Pro-Angiogenic Cells. Nutrients 2017, 9, 1328. [CrossRef] [PubMed] 
23. Russo, M.; Arigò, A.; Calabrò, M.L.; Farnetti, S.; Mondello, L.; Dugo, P. Bergamot (Citrus bergamia Risso) as a source of nutraceuticals: Limonoids and flavonoids. J. Funct. Foods 2016, 20, 10-19. [CrossRef]

24. Salerno, R.; Casale, F.; Calandruccio, C.; Procopio, A. Characterization of flavonoids in Citrus bergamia (Bergamot) polyphenolic fraction by liquid chromatography-high resolution mass spectrometry (LC/HRMS). PharmaNutrition 2016, 4, S1-S7. [CrossRef]

25. Formisano, C.; Rigano, D.; Lopatriello, A.; Sirignano, C.; Ramaschi, G.; Arnoldi, L.; Riva, A.; Sardone, N.; Taglialatela-Scafati, O. Detailed Phytochemical Characterization of Bergamot Polyphenolic Fraction (BPF) by UPLC-DAD-MS and LC-NMR. J. Agric. Food Chem. 2019, 67, 3159-3167. [CrossRef]

26. Gabetta, B.; Fuzzati, N.; Griffini, A.; Lolla, E.; Pace, R.; Ruffilli, T.; Peterlongo, F. Characterization of proanthocyanidins from grape seeds. Fitoterapia 2000, 71, 162-175. [CrossRef]

27. Baron, G.; Altomare, A.; Fumagalli, L.; Rumio, C.; Carini, M.; Vistoli, G.; Aldini, G. Development of a direct ESI-MS method for measuring the tannin precipitation effect of proline-rich peptides and in silico studies on the proline role in tannin-protein interactions. Fitoterapia 2019, 136, 104163. [CrossRef]

28. Dewanto, V.; Wu, X.; Adom, K.K.; Liu, R.H. Thermal processing enhances the nutritional value of tomatoes by increasing total antioxidant activity. J. Agric. Food Chem. 2002, 50, 3010-3014. [CrossRef]

29. Wang, H.; Cao, G.; Prior, R.L. Oxygen Radical Absorbing Capacity of Anthocyanins. J. Agric. Food Chem. 1997, 45, 304-309. [CrossRef]

30. Re, R.; Pellegrini, N.; Proteggente, A.; Pannala, A.; Yang, M.; Rice-Evans, C. Antioxidant activity applying an improved ABTS radical cation decolorization assay. Free Radic. Biol. Med. 1999, 26, 1231-1237. [CrossRef]

31. Brand-Williams, W.; Cuvelier, M.E.; Berset, C. Use of a free radical method to evaluate antioxidant activity. LWT-Food Sci. Technol. 1995, 28, 25-30. [CrossRef]

32. Scavello, F.; Zeni, F.; Tedesco, C.C.; Mensà, E.; Veglia, F.; Procopio, A.D.; Bonfigli, A.R.; Olivieri, F.; Raucci, A. Modulation of soluble receptor for advanced glycation end-products (RAGE) isoforms and their ligands in healthy aging. Aging 2019, 11, 1648-1663. [CrossRef] [PubMed]

33. Zeuner, M.T.; Vallance, T.; Vaiyapuri, S.; Cottrell, G.S.; Widera, D. Development and Characterisation of a Novel NF-кB reporter cell line for investigation of neuroinflammation. Mediat. Inflamm. 2017, 2017, 6209865. [CrossRef] [PubMed]

34. Allen, F.; Pon, A.; Wilson, M.; Greiner, R.; Wishart, D. CFM-ID: A web server for annotation, spectrum prediction and metabolite identification from tandem mass spectra. Nucleic Acids Res. 2014, 42, W94-W99. [CrossRef] [PubMed]

35. Aldini, G.; Regazzoni, L.; Pedretti, A.; Carini, M.; Cho, S.M.; Park, K.M.; Yeum, K.J. An integrated high resolution mass spectrometric and informatics approach for the rapid identification of phenolics in plant extract. J. Chromatogr. A 2011, 1218, 2856-2864. [CrossRef] [PubMed]

36. Chen, C.C.; Huang, Y.L.; Huang, F.I.; Wang, C.W.; Ou, J.C. Water-soluble glycosides from Ruta graveolens. J. Nat. Prod. 2001, 64, 990-992. [CrossRef]

37. Pacifico, S.; Piccolella, S.; Galasso, S.; Fiorentino, A.; Kretschmer, N.; Pan, S.P.; Bauer, R.; Monaco, P. Influence of harvest season on chemical composition and bioactivity of wild rue plant hydroalcoholic extracts. Food Chem. Toxicol. 2016, 90, 102-111. [CrossRef]

38. Abad-García, B.; Garmón-Lobato, S.; Berrueta, L.A.; Gallo, B.; Vicente, F. On line characterization of 58 phenolic compounds in Citrus fruit juices from Spanish cultivars by high-performance liquid chromatography with photodiode-array detection coupled to electrospray ionization triple quadrupole mass spectrometry. Talanta 2012, 99, 213-224. [CrossRef]

39. He, Y.; Li, Z.; Wang, W.; Sooranna, S.R.; Shi, Y.; Chen, Y.; Wu, C.; Zeng, J.; Tang, Q.; Xie, H. Chemical Profiles and Simultaneous Quantification of Aurantii fructus by Use of HPLC-Q-TOF-MS Combined with GC-MS and HPLC Methods. Molecules 2018, 23, 2189. [CrossRef]

40. Fujii, Y.; Tanaka, R.; Miyake, H.; Tamaru, Y.; Ueda, M.; Shibata, T. Evaluation for Antioxidative Properties of Phlorotannins Isolated from the Brown Alga Eisenia bicyclis, by the H-ORAC Method. Food Nutr. Sci. 2013, 4, 78-82. [CrossRef]

41. Mishra, K.; Ojha, H.; Chaudhury, N.K. Estimation of antiradical properties of antioxidants using DPPH assay: A critical review and results. Food Chem. 2012, 130, 1036-1043. [CrossRef]

42. Matuszewska, A.; Jaszek, M.; Stefaniuk, D.; Ciszewski, T.; Matuszewski, Ł. Anticancer, antioxidant, and antibacterial activities of low molecular weight bioactive subfractions isolated from cultures of wood degrading fungus Cerrena unicolor. PLoS ONE 2018, 13, e0197044. [CrossRef] [PubMed] 